DIFERENCIA EN CALIDAD DE VIDA EN PACIENTES CON RINITIS

ALÉRGICA CON BECLOMETASONA VS FUROATO DE MOMETASONA Y

FLUTICASONA

\author{
Autores: \\ Lilian Andrea Ballesteros Rodríguez, MD \\ Camilo Andrés Reyes Gélvez, MD
}

\author{
UNIVERSIDAD DEL ROSARIO \\ Escuela de Medicina y Ciencias de la Salud \\ Centro de Investigación en Ciencias de la Salud (CICS)
}

Bogotá, D.C., Febrero de 2016 


\title{
DIFERENCIA EN CALIDAD DE VIDA EN PACIENTES CON RINITIS ALÉRGICA CON BECLOMETASONA VS FUROATO DE MOMETASONA Y FLUTICASONA
}

\author{
Autores: \\ Lilian Andrea Ballesteros Rodríguez, MD \\ Camilo Andrés Reyes Gélvez, MD \\ Co-autores \\ Luis Jorge Mejía Perdigón, MD \\ Omar Alberto Gutiérrez Guaque, MD \\ Tutor Metodológico \\ Lina Sofía Morón Duarte, Epidemióloga \\ Tutor Temático \\ Camilo Andrés Reyes Gélvez, MD \\ Luis Jorge Mejía Perdigón, MD \\ Omar Alberto Gutiérrez Guaque, MD
}

\author{
UNIVERSIDAD DEL ROSARIO \\ Escuela de Medicina y Ciencias de la Salud \\ Centro de Investigación en Ciencias de la Salud (CICS)
}

Bogotá, D.C., Febrero de 2016 


\section{Nota de salvedad de responsabilidad institucional}

"La Universidad del Rosario no se hace responsable de los conceptos emitidos por los investigadores en su trabajo, solo velará por el rigor científico, metodológico y ético del mismo en aras de la búsqueda de la verdad y la justicia”. 


\section{Agradecimientos}

Tener la oportunidad de pertenecer a este proyecto investigación me generó muchas expectativas. Me siento muy agradecida primero con Dios por esta gran oportunidad; con mi esposo, que con su amor, paciencia y sabiduría me apoyó para sacar adelante este proyecto. Especiales agradecimientos para la Doctora Lina Morón y el Doctor Camilo Reyes, ya que gracias a su asesoría, persistencia y dedicación, este proyecto se pudo llevar a cabo. 
Tabla de contenido

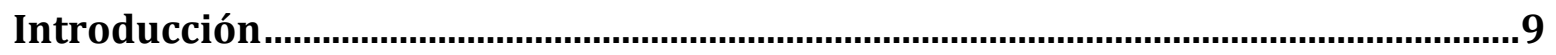

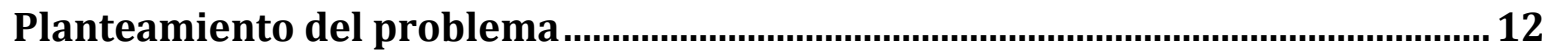

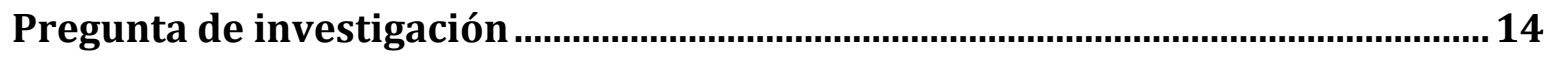

Tabla 1. Estrategia de investigación..................................................................................14

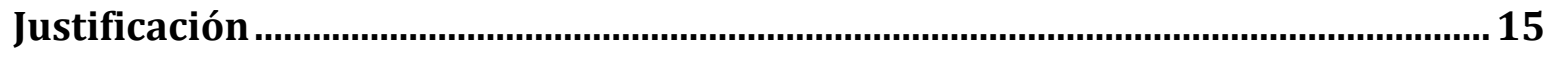

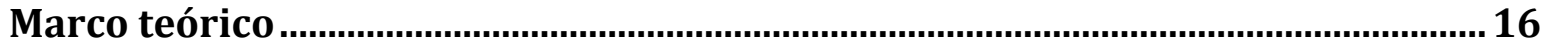

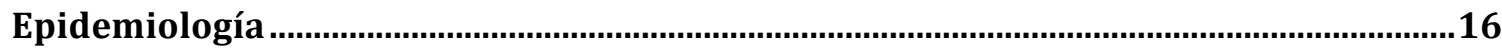

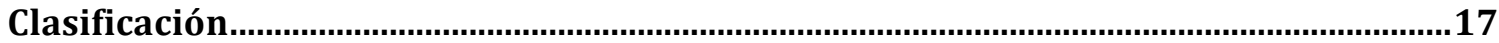

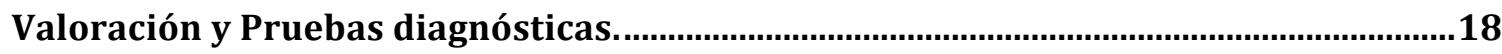

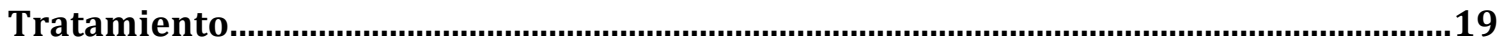

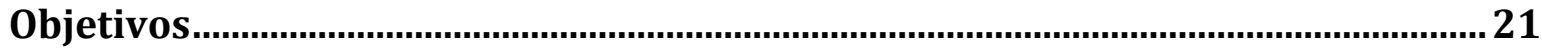

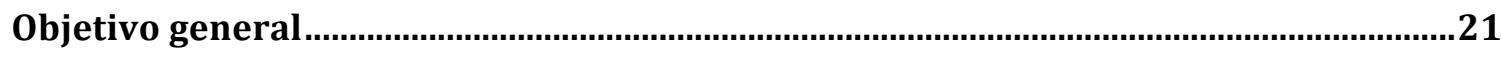

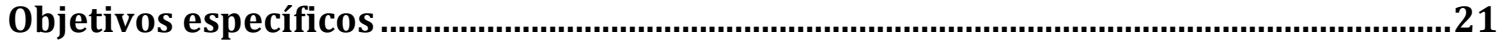

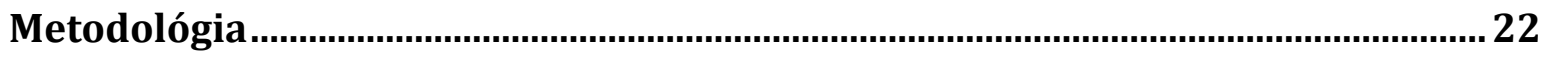

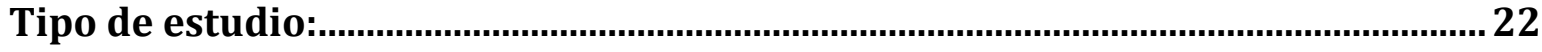

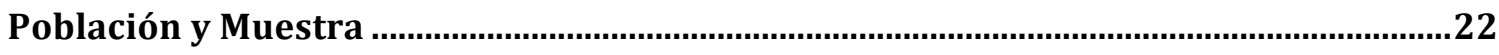

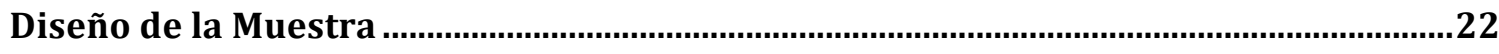

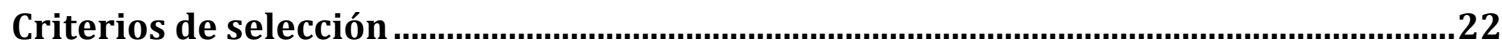

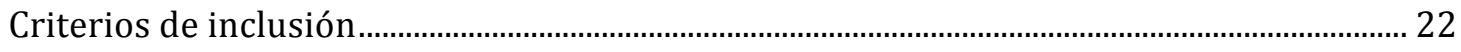

Criterios de Exclusión.................................................................................................................. 23

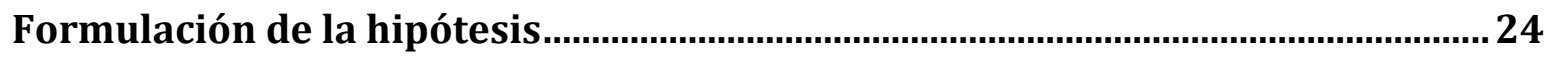

Definición y operacionalización de variables............................................................ 25

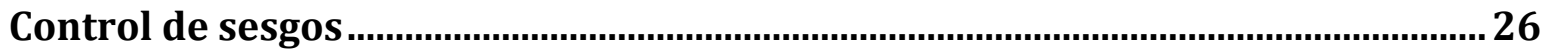


Técnicas, procedimientos e instrumentos a utilizar en la recolección de datos $\mathbf{2 6}$

Plan de procesamiento y análisis de datos ................................................................27

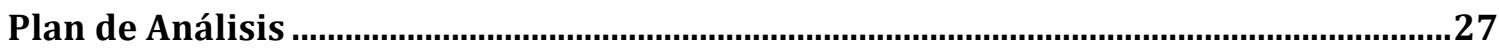

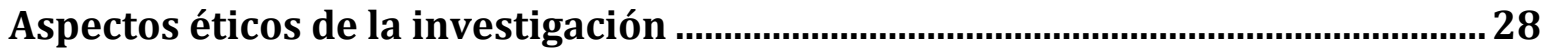

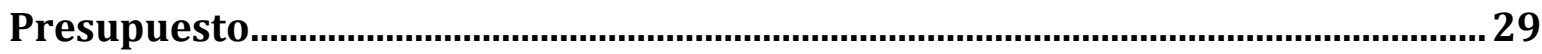

Cronograma

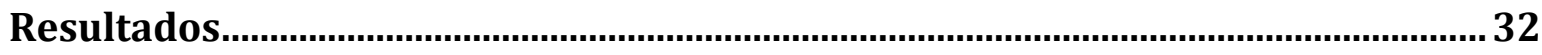

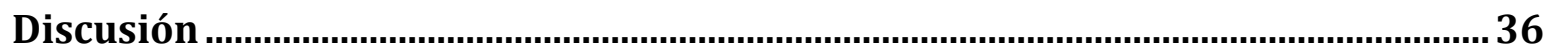

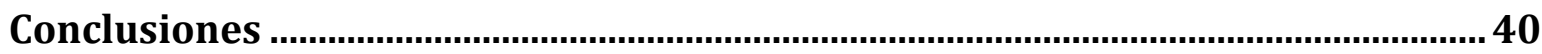

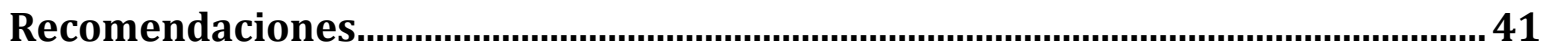

Referencias bibliográficas..................................................................................... 42

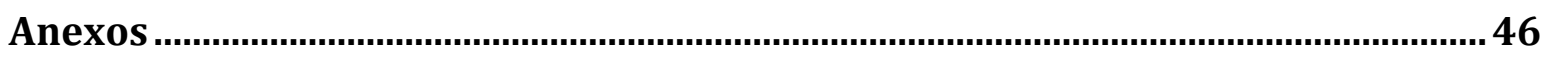




\section{Resumen:}

Introducción: La rinitis alérgica es una condición común en gran parte de la población en general. Afecta aproximadamente al 32\% de la población en general. Materiales y métodos: Estudio prospectivo en cohorte con un diagnóstico clínico de rinitis alérgica en el Hospital Universitario de La Samaritana; se evaluó el cuestionario ESPRINT-15 validado en español previo y 3 post-tratamiento con Beclometasona, Furoato de Mometasona y Fluticasona

Resultados: Se incluyeron 160 pacientes en el estudio. Previo al tratamiento, los pacientes tratados con Beclometasona y Mometasona no presentaban diferencia entre la puntuación global del cuestionario. Posterior al tratamiento, se evidenció que el grupo tratado con Furoato de Mometasona presentaba una reducción estadísticamente significativa en la severidad de síntomas (2.0 vs. 3.0, p<0.001), actividad (1.6 vs. 2.7, $\mathrm{p}<0.001)$, sueño (1.7 vs 2.9, $\mathrm{p}<0.001)$ y afectación psicológica $(1.3$ vs $2.7, \mathrm{p}<0.001)$ con respecto al grupo tratado con Beclometasona. En general, el grupo tratado con Beclometasona documentó efectos adversos más severos con respecto a la Mometasona.

Conclusiones: La Mometasona demuestra ser más efectiva y con efectos adversos menos severos con respecto a la Beclometasona, para el tratamiento de la rinitis alérgica.

Palabras clave: Rinitis alérgica, corticoide nasal, calidad de vida 


\begin{abstract}
Introduction: Allergic rhinitis is a common condition in much of the general population. It affects approximately $32 \%$ of the general population.

Materials and Methods:.Prospective cohort study with a clinical diagnosis of allergic rhinitis at the Hospital Universitario de La Samaritana; The ESPRINT-15 questionnaire validated in previous Spanish and 3 post-treatment with Beclomethasone, Mometasone Furoate and Fluticasone

Results: 160 patients were included in the study. Prior to treatment, patients treated with beclomethasone and Mometasone showed no difference between the total score of the questionnaire. After treatment, it became clear that the group treated with Mometasone group had a statistically significant reduction in the severity of symptoms (2.0 vs. 3.0, p $<0.001$ ), activity (1.6 vs. $2.7, \mathrm{p}<0.001)$, sleep (1.7 vs $2.9, \mathrm{p}<0.001)$ and psychological effects $(1.3$ vs $2.7, \mathrm{p}<0.001)$ compared to the group treated with beclomethasone. Overall, the group treated with beclomethasone document more severe adverse effects with respect to mometasone.
\end{abstract}

Conclusions: Mometasone proves to be more effective and less severe side effects compared to beclomethasone, to treat allergic rhinitis.

Keywords: allergic rhinitis, nasal corticosteroid, quality of life 


\section{Introducción}

La Rinitis Alérgica es definida como una inflamación de la mucosa nasal y es caracterizada por síntomas nasales incluyendo rinorrea anterior o posterior, estornudos, obstrucción nasal y prurito nasal, los cuales se presentan por 2 o más días consecutivos por más de 1 hora. El diagnóstico de la rinitis alérgica se basa en la concordancia entre una historia clínica típica de síntomas alérgicos y pruebas diagnósticas, como los test cutáneos alergeno específicos o la IgE total específica, sin embargo, la medición de la $\operatorname{IgE}$ total no es rutinaria para el diagnóstico de rinitis alérgica, por lo tanto, no es necesario practicar pruebas paraclínicas en la mayoría de los casos $\left(^{1}\right)$. Es una enfermedad común y prevalente, la cual afecta aproximadamente al 32\% de la población Colombiana $\left({ }^{2}\right)$. Genera una importante carga socioeconómica y tiene un impacto significativo en la calidad de vida, afectando principalmente su componente social de acuerdo a la actualización en Rinitis Alérgica y su impacto en el Asma (ARIA) del 2008 ( $\left.{ }^{1}\right)$. El grado en el cual la calidad de vida es comprometida, varía principalmente dependiendo del estándar que un individuo considere con respecto a "Buen estado de salud". Los cuestionarios enfermedad específicos (Sinonasal Outcome Test, SNOT-22, Rhinitis Quality of Life Questionnaire, the Rhinosinusitis Disability Index, ESPRINT questionnaire) son instrumentos ampliamente usados para medir la calidad de vida en diferentes aspectos, porque describen de manera más precisa los problemas asociados con la enfermedad y ayudan a determinar la eficacia de un tratamiento.

Con respecto a su tratamiento, los corticoides tópicos nasales son unos de los medicamentos más comunmente usados para el control de esta entidad. Estos medicamentos son altamente efectivos y sus efectos adversos son pocos y escasamente 
significativos; por lo tanto son considerados por muchos, como la primera línea de tratamiento para la rinitis alérgica y no alérgica. En relación al Plan Obligatorio de Salud (POS), el único corticoide nasal incluido para el tratamiento de la rinitis alérgica es la Beclometasona Dipropionato. Sin embargo, existen otros corticoides nasales usados en nuestro medio, como la Budesonida, la Mometasona, la Fluticasona, Triamcinolona y la Ciclesonida, los cuales no están incluidos en el POS y su adquisición es asumida por el paciente. En general, todos tienen el mismo efecto farmacológico, sin embargo, hay diferencias entre ellos. La Fluticasona tiene el doble de potencia que la Beclometasona $\left({ }^{3}\right)$. La Fluticasona y la Mometasona tienen metabolismo de primer paso hepático, debido a su absorción por el sistema gastrointestinal, lo cual evita su absorción sistémica, donde la Fluticasona ha mostrado que tiene una alta capacidad para adherirse al tejido nasal, y por ende, una baja absorción sistémica. La biodisponibilidad de los corticoides intranasales es variable para cada molécula; para la Mometasona y la Fluticasona es menor al 1\%, para la Beclometasona es de un 40-50\% lo que puede influir en el desarrollo de efectos adversos. Un único estudio ha reportado un leve efecto en el crecimiento de niños recibiendo una dosis estándar de Beclometasona durante 1 año de tratamiento $\left({ }^{4}\right)$. La resequedad nasal, formación de costras, epistaxis, y la cefalea entre otros, son los efectos adversos más comúnmente encontrados. De estos, la irritación nasofaríngea se presenta principalmente con el uso de la Beclometasona hasta en un $24 \%$ de los pacientes, lo cual ha llevado a muchos Otorrinolaringólogos a formular otros corticoides nasales como Furoato de Mometasona o Fluticasona.

En Colombia se han realizado estudios de costo-efectividad con el uso corticoides nasales tópicos para el tratamiento del asma debido a su alta prevalencia e incidencia, sin embargo, son muy pocos o nulos los estudios realizados de costo-efectividad con el uso de corticoides nasales para el tratamiento de la rinitis alérgica en adultos.

El propósito del presente trabajo es comparar la diferencia en la calidad de vida mediante el cuestionario ESPRINT-15 validado al español en pacientes con rinitis alérgica tratados independientemente con Beclometasona, Furoato de Mometasona y Fluticasona en el Hospital Universitario de la Samaritana durante el período 2013-2015. 
En la actualidad se encuentra un estudio realizado en el 2015, donde se desarrolló un modelo de costo-efectividad, eficacia y efectos adversos con el uso de Furoato de Mometasona Vs Beclometasona para el tratamiento de pacientes pediátricos con rinitis alérgica durante 12 meses, este análisis concluyó que en comparación con el uso de Beclometasona, la terapia con Furoato de Mometasona se asoció con costos más bajos (US \$229,78 VS \$ 289.74 coste medio por paciente de más de 12 meses). Dentro de los costos incluidos en este estudio se incluyen todos lo efectos adversos presentados por los pacientes con el uso de Beclometasona y su tratamiento respectivo. En comparación con el uso de Furoato de Mometasona se evidencia la eficacia del tratamiento y la baja incidencia de efectos adversos presentados por los pacientes. (9)

Los cuestionarios enfermedad específicos (Sinonasal Outcome Test, SNOT-22, Rhinitis Quality of Life Questionnaire, the Rhinosinusitis Disability Index, ESPRINT-15 valido al español) son instrumentos ampliamente usados para medir la calidad de vida, porque describen de manera más precisa los problemas asociados con la enfermedad y ayudan a determinar la eficacia de un tratamiento $\left({ }^{1}\right)$; cabe aclarar que el cuestionario utilizado por nuestro estudio fue definido por sociedad española de alergología e inmunologia clínica. 


\section{Planteamiento del problema}

En Colombia, el tratamiento con corticoides nasales para pacientes con rinitis alérgica, está limitado al único medicamento incluido en el Plan Obligatorio de Salud (Beclometasona), la experiencia de cada profesional y a su preferencia a determinada molécula. Los pacientes que no responden al tratamiento o que presenten reacciones indeseables con el uso de la Beclometasona, le son formulados corticoides nasales como la Fluticasona, Furoato de Mometasona, Budesonida, Triamcinolona o la Ciclesonida; los cuales no están incluidos en el POS, y por lo tanto los costos de estos medicamentos son asumidos por el paciente. A pesar que es una enfermedad de alta prevalencia en nuestro país, se conoce muy poco las diferencias en la calidad de vida de pacientes con diagnóstico clínico de rinitis alérgica tratados con Beclometasona, Mometasona, Fluticasona y Budesonida. Esta es la razón por la cuál, el propósito del presente trabajo es comparar la diferencia en calidad de vida mediante el cuestionario ESPRINT -15 validado al español, para pacientes con Rinitis Alérgica, tratados independientemente con estos corticoides nasales, para de esta forma determinar con cuál de ellos el paciente obtiene más beneficio y comprobar así como mejora su percepción de su calidad de vida. Adicionalmente, en nuestro estudio buscamos determinar la proporción de los efectos adversos con el uso de corticoides nasales (Beclometasona, Furoato de Mometasona y Fluticasona) debido a que en Colombia esto a motivado a realización

de diversas publicaciones con respecto a los costos adicionales que genera los efectos adversos secundarios presentados por el uso de corticoide nasal incluido dentro del POS. Esto es muy importante para nuestro ejericio profesional como 
Otorrinolaringólogos ya que podremos tener una visión más objetiva en el momento de tomar una decisión clínica con respecto a la instauración del tratamiento de la rinitis alérgica. 


\section{Pregunta de investigación}

En la presente investigación se tuvieron en cuenta los siguientes aspectos para el desarrollo de la pregunta:

- Problema que motivó la investigación: Evaluar la calidad de vida y los efectos indeseables en pacientes con rinitis alérgica tratados con corticoide nasal.

- Intervención utilizada: Comparación de Corticoide nasal incluido en el POS (Beclometasona) y el no incluido en el POS (Furoato de Mometasona, Fluticasona)

- Desenlace: Se espera que el tratamiento con corticoide nasal Furoato de mometasona y fluticasona presente mejores resultados con respecto a puntajes de cuestionario SPRINT-15 y efectos indeseables.

- Tipo de estudios que aportan la mejor información para la técnica de análisis utilizada: Ensayos clínicos controlados aleatorizados (ECCA), estudios de casos y controles y de cohorte. La estrategia utilizada (estrategia PICO) para construir la pregunta de investigación es la siguiente:

\section{Tabla 1. Estrategia de investigación}

\begin{tabular}{|l|l|l|}
\hline P & Población. & Adultos mayores de 18 años \\
\hline I & Intervención & Corticoides nasales \\
\hline C & Comparación & $\begin{array}{l}\text { Beclometasona dipropionato vs Furoato de Mometasona vs } \\
\text { Fluticasona }\end{array}$ \\
\hline O & $\begin{array}{l}\text { Resultado } \\
\text { (Outcomes) }\end{array}$ & $\begin{array}{l}\text { Mejoría de la percepción de la calidad y efectos indeseables con } \\
\text { corticoides nasales }\end{array}$ \\
\hline
\end{tabular}




\section{Justificación}

En Colombia hay muy pocos estudios acerca de cuál es la afectación en la calidad de vida de los pacientes con rinitis alérgica tratados con corticoide nasal. Se conoce poco si existen diferencias en la calidad de vida de pacientes con diagnóstico clínico de rinitis alérgica tratados con Beclometasona, Furoato de Mometasona, Fluticasona. Esta es la razón por la cuál el propósito del presente trabajo es comparar la diferencia en la calidad de vida de los pacientes utilizando el cuestionario ESPRINT -15 validado al español, para pacientes con rinitis alérgica tratados independientemente con estos corticoides nasales. Adicionalmente, buscamos determinar la proporción de los efectos adversos con el uso de corticoides nasales (Beclometasona, Furoato de Mometasona y Fluticasona), para de esta forma determinar con cuál de ellos el paciente obtiene más beneficios y comprobar así cómo mejora la percepción de su calidad de vida. 


\section{Marco Teórico}

La rinitis es caracterizada por 1 o más episodios de los siguientes síntomas: congestión nasal, rinorrea (anterior y posterior), estornudos y prurito nasal. Como en el asma, una respuesta temprana y una respuesta tardía han sido descritas en pacientes con rinitis

alérgica. Ambas fases están caracterizadas por síntomas como estornudos, rinorrea y congestión nasal, sin embargo, la congestión nasal es predominantemente una respuesta de la fase tardía. Es una causa significativa de morbilidad, costo en tratamiento médico, disminución en la productividad laboral llevando a incapacidad médica, afectando la calidad de vida del paciente, la cual puede estar asociada con condiciones como fatiga, cefalea, compromiso cognoscitivo y alteraciones del sueño.

\section{Epidemiología}

La rinitis alérgica afecta entre el $10-30 \%$ de la población adulta y hasta el $40 \%$ de la población pediátrica $\left(^{5}\right)$. La prevalencia de la rinitis alérgica estacional es alta en niños y adolescentes, donde la rinitis alérgica perenne tiene una mayor prevalencia en adultos $\left({ }^{6}\right)$. Se debe clasificar de acuerdo a su etiología en alérgica y no alérgica, y debe ser diferenciada de condiciones que simulan los síntomas de la rinitis. La rinitis estacional es causada por la reacción mediada por IgE a aero-alergenos estacionales. La rinitis alérgica perenne es causada por la reacción mediada por IgE a aero-alergenos del medio ambiente, como polvo, hongos, alérgenos animales, alérgenos ocupacionales, polen etc. Los factores de riesgo para rinitis incluyen: 1) Antecedente familiar para atopia, 2) $\operatorname{IgE}$ en suero superior a $100 \mathrm{UI} / \mathrm{mL}$ antes de $\operatorname{los} 6$ años, 3) Estrato socioeconómico alto y 4) La presencia de prueba cutánea positiva para alergia. La sensibilización raramente comienza antes de los 6 meses de edad, pero puede iniciar entre los 6 meses y los 2 años de vida $\left({ }^{7}\right)$. 
Los costos para el tratamiento de rinitis alérgica y los costos indirectos relacionados a las alteraciones en productividad laboral como resultado de la enfermedad son substanciales. El costo estimado total directo ( 7.3 billones de dólares) e indirecto (4.28 billones de dólares) para los Estados Unidos de América en el 2002, siendo el total de 11.58 billones $\left({ }^{7}\right)$. Existe varios tipos de clasificación de la rinitis alégica según su factor etiológico, frecuencia y severidad de los síntomas, esta última es según las guías ARIA.

\section{Clasificación basada en etiología}

- Rinitis No alérgica: Es caracterizada por síntomas periódicos que no son el resultado de eventos dependientes de IgE.

- Rinitis vasomotora: hace parte de un grupo heterogéneo de pacientes con síntomas nasales crónicos que no son inmunológicos o infecciosos en origen y usualmente no están asociados con eosinofilia nasal.

- Rinitis por comida y alcohol: La rinitis se puede presentar posterior a la ingesta de comida o alcohol. Esto puede estar producido por estimulación vagal, vasodilatación nasal, alergia a la comida y otros mecanismos no identificados.

- Rinitis infecciosa: La Rinitis aguda infecciosa es usualmente el resultado de uno a un largo número de virus, sin embargo, la infección bacteriana secundaria con compromiso sinusal puede ser una complicación. Las infecciones virales son responsables de aproximadamente el 98\% de las rinitis infecciosas agudas y de la mayoría de los síntomas riníticos en los niños jóvenes $\left(^{7}\right)$.

- Rinitis Ocupacional: Esta rinitis es ocasionada por sustancias volátiles en el medio ambiente laboral, la cual puede estar mediada por factores alérgicos y no alérgicos. En ocasiones coexiste con asma ocupacional.

- Rinitis Hormonal: Las causas de rinitis hormonal incluyen el embarazo y la rinitis asociada al ciclo menstrual. La rinitis en el embarazo se presenta después del 2 do mes de embarazo, y usualmente desaparece a las 2 semanas del post-parto.

- Rinitis inducida por medicamentos: Puede ser ocasionada por una variedad de medicamentos, incluyendo los Inhibidores de la encima convertidora de angiotensina (IECA), inhibidores selectivos de la 5-fosfodiesterasa, la fentolamina, 
antagonistas de receptores alfa, asa y otros anti-inflamatorios no esteroideos (AINES). La rinitis medicamentosa es un síndrome de rebote al sobreuso de los descongestionantes alfa adrenérgicos intranasales o la cocaína.

- Rinitis atrófica: Puede ser primaria o secundaria resultando en la formación de costras y cacosmia.

\section{Clasificación basada en frecuencia y severidad de síntomas según consenso ARIA}

Según la frecuencia, se clasifica en :

- Rinitis alérgica intermitente: cuando los síntomas se presentan menos de cuatro días a la semana o por menos de cuatro semanas consecutivas.

- Rinitis alérgica persistente: cuando los síntomas se presentan por más de cuatro días a la semana o más de cuatro semanas consecutivas

De acuerdo con la severidad de los síntomas y su impacto en la calidad de vida de los pacientes (alteraciones en el sueño, cambios emocionales, interferencia con el trabajo o estudio, etc.) se clasifica en:

- Leve: los síntomas no interfieren en la vida del paciente.

- Moderada/severa: los síntomas alteran la calidad de vida del paciente.

La poliposis nasal, las obstrucciones mecánicas como la desviación septal, la hipertrofia de los cornetes nasales, la rinoliquia y la disfunción ciliar hacen parte de los diagnósticos diferenciales.

\section{Valoración y Pruebas diagnósticas.}

La valoración adecuada de pacientes con rinitis incluye: una determinación del patrón, cronicidad y estacionalidad de los síntomas nasales; la respuesta a medicamentos, la presencia de condiciones coexistentes, exposición ocupacional; y determinar el medio ambiente en el que se desenvuelve el paciente e identificar factores precipitantes. La evaluación de pacientes riníticos debe incluir la valoración de la calidad de vida (Recomendación C) $\left(^{7}\right)$. 
El exámen físico se debe realizar basándose en los órganos que afecta la rinitis. El exámen físico ayuda, sin embargo, no establece el diagnóstico de rinitis.

Determinar IgE específico, preferiblemente mediante pruebas cutáneas, es indicado para proporcionar evidencia de una base alérgica para los síntomas del paciente, para confirmar o excluir causas de los síntomas de paciente o establecer que alérgenos el paciente debe evitar.

Los frotis nasales de rutina no son necesarios para el diagnóstico de rinitis alérgica cuando el diagnóstico es claro mediante historia clínica, el examen físico e $\operatorname{IgE}$ específica, sin embargo, son útiles cuando el diagnóstico es dudoso $\left({ }^{7}\right)$.

\section{Tratamiento}

El manejo apropiado de la rinitis puede ser un componente importante en el manejo efectivo de condiciones respiratorias coexistentes, como asma, sinusitis y apnea del sueño.

Manejo del medio ambiente, control de la humedad, especialmente para el control de ácaros. El mejor tratamiento para la rinitis exacerbada por irritantes, como el tabaco y el formaldehido, es evitar su exposición.

Los antihistiamínicos de segunda generación son preferidos a los de primera generación, ya que estos, producen sedación, compromiso del desempeño funcional y tienen efectos anticolinérgicos. Entre los nuevos, antihistamínicos no sedativos, ninguno ha obtenido de manera concluyente una tasa superior de respuesta global.

Los antihistamínicos intranasales pueden considerarse como primera línea de tratamiento para rinitis alérgica y no alérgica, ya que se consideran igual o superiormente efectivos a los antihistamínicos orales de segunda generación. Se ha considerado que tienen un efecto clínicamente significativo sobre la congestión nasal, sin embargo, se consideran menos efectivos que los corticoesteroides intranasales.

Los descongestionantes orales, como la pseudoefedrina (retirada en Colombia) y fenilefrina, son agonistas alfa adrenérgicos que pueden reducir la congestión nasal, no obstante, produce efectos adversos como insomnio, irritabilidad y palpitaciones. Se deben 
usar por corto tiempo y posiblemente de manera intermitente, debido al riesgo de desarrollar rinitis medicamentosa. Los medicamentos antigripales de venta libre, deben ser evitados en pacientes menores de 6 años debido a su potencial efecto tóxico.

Los corticoesteroides intranasales son los medicamentos más efectivos para el control de los síntomas en rinitis alérgica (Recomendación A). Se ha visto que son más efectivos que la combinación de antihistamínicos y antileucotrienos en el tratamiento de rinitis estacional. Aunque son efectivos cuando se usan según necesidad, se ha demostrado, que no tienen el mismo efecto que cuando se usan de forma regular. Cuando se usan a dosis adecuadas, generalmente no produce efectos adversos. Los efectos adversos locales son mínimos, la irritación nasal o epistaxis se pueden $\operatorname{presentar}\left({ }^{7}\right)$.

Un ciclo corto de corticoides orales (5-7 días) puede ser apropiado para el control de sintomatología severa e intratable o para el manejo de la poliposis nasal.

El cromoglicato de sodio es efectivo en algunos pacientes para la prevención y el tratamiento de rinitis alérgica y está asociado a efectos secundarios mínimos. Sin embargo, es menos efectivo que los corticoides nasales y no se ha estudiado en comparación a antagonistas de leucotrienos y antihistamínicos. Los anticolinérgicos intranasales son útiles para la rinorrea, sin embargo, no tienen efecto sobre otros síntomas nasales. Se han usado en conjunto con los corticoides nasales demostrando mayor efectividad que cuando se administran por separado.

Los antihistamínicos orales y nasales, lo antileucotrienos, los corticoides intranasales y la inmunoterapia son tratamientos para rinitis alérgica que han reportado mejoría asociada a los síntomas alérgicos oculares en estudios controlados.

El omalizumab, un inhibidor de de receptores de $\operatorname{IgE}$, ha demostrado efectividad en rinitis alérgica, sin embargo, la FDA lo ha autorizado únicamente para la asma alérgica.

Es fundamental manejar la rinitis alérgica, debido a que mejoran el control del asma y reducen el riesgo para desarrollar asma. 


\section{Objetivos}

\section{Objetivo general:}

- Comparar la diferencia en la calidad de vida mediante el cuestionario ESPRINT-15 validado al español en pacientes con rinitis alérgica tratados independientemente con Beclometasona, Furoato de Mometasona y Fluticasona en el Hospital Universitario de la Samaritana durante el período 2013-2015.

\section{Objetivos específicos:}

1. Caracterizar la muestra de estudio en cuanto a variables epidemiológicas.

2. Calcular la diferencia del promedio y puntuación global antes y después del tratamiento con (Beclometasona, Furoato de Mometasona y Fluticasona) de cada uno de los ítems: Síntomas, actividad, sueño y afectación psicológica contenidos en el cuestionario ESPRINT-15.

3. Evaluar la percepción en estado de salud de los pacientes con Rinitis Alérgica previo y posterior al tratamiento con corticoides nasales a través del cuestionario ESPRINT-15.

4. Determinar la proporción de los efectos adversos con el uso de corticoides nasales (Beclometasona, Furoato de Mometasona y Fluticasona) 


\section{Metodología}

\section{Tipo de estudio:}

Estudio antes y después en una cohorte prospectivo de pacientes con rinitis alérgica.

\section{Población y Muestra:}

Población o universo: Pacientes con rinitis alérgica que acudieron a la consulta externa en el Hospital Universitario de la Samaritana en el año 2013-2015.

Marco Muestral: Paciente con rinitis alérgica que acudieron a la consulta externa en el Hospital Universitario de la Samaritana en el período comprendido entre 2013- 2015 y que cumplieran con los criterios de selección definidos en el estudio.

\section{Diseño de la Muestra}

En el Hospital Universitario de la Samaritana, el promedio semanal de pacientes con rinitis alérgica atendidos en la consulta es de 25 pacientes, es decir que al mes serían aproximadamente $100 \mathrm{y}$ al año un total de 1300 pacientes. De esa población total, teniendo en cuenta un I.C de 95\%, un error del 5\% y una prevalencia estimada de rinitis alérgica para Colombia del 32\% $\left(^{2}\right)$, la muestra sería de 160 . Estos pacientes fueron dividos aleatoriamente en tres grupos tratados con Beclometasona 2 puff en cada fosa nasal cada 12 horas, Furoato de Mometasona 2 puff en cada fosa nasal cada día y Fluticasona 2 puff en cada fosa nasal cada día y clasificados según el consenso ARIA

\section{Criterios de selección}

\section{Criterios de inclusión:}

- Pacientes mayores de 18 años de edad.

- Pacientes que refieran 1 o más episodios de congestión nasal, rinorrea (anterior y posterior), estornudos y prurito nasal.

- Pacientes que cumplan los criterios clínicos y paraclínicos de Rinitis Alérgica definidos por el consenso de la ARIA.

- Voluntarios que acepten y firmen el consentimiento informado. 
- Voluntarios que diligencien el cuestionario de calidad de vida ESPRINT-15 validado al español.

- Pacientes con ASMA y/o Dermatitis Atópica con diagnóstico de Rinitis Alérgica definidos por el consenso de la ARIA.

\section{Criterios de Exclusión}

- Pacientes que estén recibiendo antihistamínicos orales o nasales, descongestionantes nasales, cromoglicato de sodio intranasal, anticolinérgicos intranasales, omalizumab, corticoides sistémicos, antileucotrienos o terapia inmunológica.

- Pacientes que estén recibiendo medicamentos homeopáticos.

- Pacientes embarazadas.

- Pacientes que abandonen el estudio. 


\section{Formulación de la hipótesis}

Hipótesis Nula: No existen diferencias en la calidad de vida de pacientes con rinitis alérgica y no alérgica tratados con los corticoides nasales Beclometasona, Furoato de Mometasona, Fluticasona.

Hipótesis Alternativa: Existen diferencias en la calidad de vida de pacientes con rinitis alérgica y no alérgica tratados con los corticoides nasales Beclometasona, Furoato de Mometasona, Fluticasona. 


\section{Definición y operacionalización de variables}

\begin{tabular}{|c|c|c|c|c|c|c|c|}
\hline & $\begin{array}{l}\text { 1: No me } \\
\text { ha } \\
\text { molestado } \\
\text { nada }\end{array}$ & $\begin{array}{l}\text { 2: No me } \\
\text { ha } \\
\text { molestado } \\
\text { casi nada }\end{array}$ & $\begin{array}{c}\text { 3: Me ha } \\
\text { molestado } \\
\text { un poco }\end{array}$ & $\begin{array}{c}\text { 4: Me ha } \\
\text { molestado } \\
\text { moderada- } \\
\text { mente }\end{array}$ & $\begin{array}{l}\text { 5: Me ha } \\
\text { molestado } \\
\text { bastante }\end{array}$ & $\begin{array}{c}\text { 6: Me ha } \\
\text { molestado } \\
\text { mucho }\end{array}$ & $\begin{array}{c}\text { 7: Me ha } \\
\text { molestado } \\
\text { muchísimo }\end{array}$ \\
\hline \multicolumn{8}{|l|}{ SÍNTOMAS } \\
\hline \multicolumn{8}{|l|}{$\begin{array}{l}\text { La sensación de tener la nariz tapada o la } \\
\text { sensación de obstrucción }\end{array}$} \\
\hline \multicolumn{8}{|l|}{ La mucosidad nasal liquida o como agua } \\
\hline \multicolumn{8}{|l|}{ El picor en la nariz o estornudos repetidos } \\
\hline \multicolumn{8}{|l|}{ El picor de ojos o tener que rascarse los ojos } \\
\hline \multicolumn{8}{|l|}{$\begin{array}{l}\text { La dificultad para respirar, la sensación de } \\
\text { asfixia o ahogo }\end{array}$} \\
\hline \multicolumn{8}{|l|}{ ACTIVIDADES DE LA VIDA DIARIA } \\
\hline \multicolumn{8}{|l|}{$\begin{array}{l}\text { La incomodidad o dificultad para trabajar, a } \\
\text { causa de la rinitis. }\end{array}$} \\
\hline \multicolumn{8}{|l|}{$\begin{array}{l}\text { Los síntomas de la rinitis cenando o tomando } \\
\text { algo fuera de casa. }\end{array}$} \\
\hline \multicolumn{8}{|l|}{$\begin{array}{l}\text { Interrumpir constantemente lo que está } \\
\text { haciendo, a causa de la Rinitis }\end{array}$} \\
\hline \multicolumn{8}{|l|}{ SUEÑO } \\
\hline \multicolumn{8}{|l|}{$\begin{array}{l}\text { Los problemas para dormir o las dificultades } \\
\text { para conciliar el sueño, a causa de la rinitis }\end{array}$} \\
\hline \multicolumn{8}{|l|}{$\begin{array}{l}\text { Levantarse con sequedad de boca o } \\
\text { despertarse por esto, a causa de la rinitis }\end{array}$} \\
\hline \multicolumn{8}{|l|}{ Dormir mal, a causa de la rinitis } \\
\hline \multicolumn{8}{|l|}{ AFECTACIÓN PSICOLÓGICA } \\
\hline \multicolumn{8}{|l|}{ Tener que estar pendiente de la rinitis } \\
\hline \multicolumn{8}{|l|}{$\begin{array}{l}\text { Estar más irritable o de mal humor, a causa de } \\
\text { la rinitis }\end{array}$} \\
\hline Pasarlo o sentIrse mal, a causa de la rinitis & & & & & & & \\
\hline $\begin{array}{l}\text { Percepción de la enfermedad - estado de } \\
\text { salud por parte del paciente }\end{array}$ & Excelente: 0 & $\begin{array}{c}\text { Muy } \\
\text { buena:1 }\end{array}$ & Buena: 2 & Regular: 3 & Mala: 4 & & \\
\hline
\end{tabular}




\section{Control de sesgos}

Sesgos de selección: Se incluyeron en el estudio estrictamente a los pacientes que cumplan con los criterios de inclusión/exclusión los cuales fueron definidos anteriormente.

Sesgo de interpretación de la escala de calidad de vida: El cuestionario utilizado para valorar la calidad de vida se encuentra validado y su interpretación se realizó con la metodología definida por dicho cuetionario.

Sesgo de información: La información se tomó de la fuente primaria y el mismo paciente.

\section{Técnicas, procedimientos e instrumentos a utilizar en la recolección de datos}

Para la recolección de los datos se aplicó el cuestionario ESPRINT-15 validado al español

$\left({ }^{8}\right)$, el cual fue diligenciado por el paciente al momento de ser incluido en el estudio. Se diligenció otro cuestionario a los 3 meses cuando el paciente fue valorado en el control médico.

Se construirá una base de datos en Excel versión 2007 en donde se incluirán los ítems del cuestionario ESPRINT 15; la depuración y procesamiento se realizó con el paquete estadístico SPSS 20. 


\section{Plan de procesamiento y análisis de datos}

\section{Plan de Análisis}

El análisis de los ítems o dominios se realizó de acuerdo a la metodología definida por el mismo cuestionario en donde se promediarán los ítems (0-6). Adicionalmente se obtuvo una suma global de las puntuaciones de los 14 ítems, más la puntuación dada en el cuestionario general. Esto se dividió por el número total de ítems.

Se realizó una comparación de medias para datos relacionados entre la puntuación por dimensiones y la puntuación global, comparando los resultados obtenidos en el cuestionario de antes del tratamiento y posterior al tratamiento para determinar las diferencias existentes en la calidad de vida de pacientes con rinitis alérgica y no alérgica tratados con los corticoides nasales Beclometasona, Furoato de Mometasona, Fluticasona. Se utilizó la prueba específica según el caso, t de Student o wilconxon según el comportamiento de normalidad de los datos para muestras relacionadas, se consideró como estadísticamente significativo una $\mathrm{p}<0.05$, con un intervalo de confianza del $95 \%$. 


\section{Aspectos éticos de la investigación}

El presente trabajo realizó de acuerdo a las normas establecidas por la Resolución No. 008430 de 1993 del Ministerio de Salud de Colombia que regula este tipo de investigaciones. De acuerdo a la resolución se clasifica este trabajo como Investigación de riesgo mínimo, debido a que se emplean procedimientos terapéuticos rutinarios con medicamentos de uso común de acuerdo a las indicaciones, dosis y vía de administración establecidas. Se anexa carta de aprobación del Centro de Investigaciones del Hospital Universitario la Samaritana. (Anexo 4) 


\section{Presupuesto}

\begin{tabular}{|c|c|c|c|c|c|c|c|}
\hline \multicolumn{8}{|l|}{ PRESUPUESTO } \\
\hline \multicolumn{4}{|c|}{ CATEGORIA } & \multirow{2}{*}{$\begin{array}{c}\text { COSTO } \\
\text { UNITARIO }\end{array}$} & \multirow{2}{*}{$\begin{array}{l}\text { PRESUPUESTO } \\
\text { REQUERIDO }\end{array}$} & \multirow{2}{*}{$\begin{array}{c}\text { PRESUPUESTO } \\
\text { POR } \\
\text { FINANCIAR } \\
\text { POR EL } \\
\text { HOSPITAL } \\
\end{array}$} & \multirow{2}{*}{$\begin{array}{c}\text { JUSTIFICACIÓN } \\
\text { DEL } \\
\text { PRESUPUESTO }\end{array}$} \\
\hline PERSONAL & $\begin{array}{c}\text { \% DE } \\
\text { TIEMPO } \\
\text { DEDICADO }\end{array}$ & $\begin{array}{c}\text { Días / } \\
\text { Persona }\end{array}$ & Cantidad & & & & \\
\hline \multirow[t]{3}{*}{ 1. Investigadores principales } & $30 \%$ & $\begin{array}{l}\text { Camilo } \\
\text { Reyes }\end{array}$ & 1 & 768.000 & 768.000 & 0 & $\begin{array}{l}\text { Camilo Andres } \\
\text { Reyes Gelves }\end{array}$ \\
\hline & $30 \%$ & $\begin{array}{l}\text { Camilo } \\
\text { Reyes }\end{array}$ & 1 & 768.000 & 768.000 & 0 & $\begin{array}{l}\text { Camilo Andres } \\
\text { Reyes Gelves }\end{array}$ \\
\hline & $30 \%$ & $\begin{array}{l}\text { Camilo } \\
\text { Reyes }\end{array}$ & 1 & 768.000 & 768.000 & 0 & $\begin{array}{l}\text { Camilo Andres } \\
\text { Reyes Gelves }\end{array}$ \\
\hline Papelería & & & 1 & 100.000 & 100.000 & 0 & Papelería \\
\hline
\end{tabular}

\begin{tabular}{|c|c|c|c|c|c|c|c|}
\hline EQUIPO: & & & & & & & \\
\hline 1. Computador & $100 \%$ & NA & 1 & 600.000 & 600.000 & 0 & \\
\hline 1. Estadísticos & $100 \%$ & Lina Morón & 1 & 800.000 & 800.000 & 0 & $\begin{array}{l}\text { Análisis estadístico } \\
\text { de los resultados }\end{array}$ \\
\hline \multicolumn{8}{|l|}{ CORTICOIDE NASAL: } \\
\hline Beclometasona & NA & 90 días & 1 & NA & $\begin{array}{c}\text { Asumido por el } \\
\text { Plan Obligatorio } \\
\text { de Salud }\end{array}$ & 0 & \begin{tabular}{|l} 
Corticoide nasal \\
incluido en el Plan \\
Obligatorio de \\
Salud, indicado \\
para el manejo de \\
la sintomatología \\
nasal de la rinitis \\
alérgica.
\end{tabular} \\
\hline Furoato de Mometasona & NA & 90 días & 1 & & $\begin{array}{c}\text { Asumido por el } \\
\text { paciente }\end{array}$ & 0 & $\begin{array}{l}\text { Corticoide anti- } \\
\text { inflamatorio nasal } \\
\text { indicado para el } \\
\text { manejo de la } \\
\text { sintomatología } \\
\text { nasal de la rinitis } \\
\text { alérgica }\end{array}$ \\
\hline Furoato de Fluticasona & NA & 90 días & 1 & & $\begin{array}{c}\text { Asumido por el } \\
\text { paciente }\end{array}$ & 0 & $\begin{array}{l}\text { Corticoide anti- } \\
\text { inflamatorio nasal } \\
\text { indicado para el } \\
\text { manejo de la } \\
\text { sintomatología } \\
\text { nasal de la rinitis } \\
\text { alérgica }\end{array}$ \\
\hline Budesonida & NA & 90 días & 1 & & $\begin{array}{c}\text { Asumido por el } \\
\text { paciente }\end{array}$ & 0 & $\begin{array}{l}\text { Corticoide anti- } \\
\text { inflamatorio nasal } \\
\text { indicado para el } \\
\text { manejo de la } \\
\text { sintomatología } \\
\text { nasal de la rinitis } \\
\text { alérgica }\end{array}$ \\
\hline Co-Autor & $30 \%$ & $\begin{array}{l}\text { Liliana } \\
\text { Ballesteros } \\
\text { Rodríguez }\end{array}$ & 1 & 3.860 .000 & 1.158 .000 & 0 & Asesoría \\
\hline
\end{tabular}




\begin{tabular}{|c|c|c|c|c|c|c|c|}
\hline Co-Autor & $30 \%$ & $\begin{array}{l}\text { Omar } \\
\text { Gutiérrez }\end{array}$ & 1 & 2.500 .000 & 750.000 & 0 & Asesoría \\
\hline $\begin{array}{l}\text { GASTOS DE } \\
\text { DIVULGACIÓN }\end{array}$ & & & & $\$ 0.00$ & $\$ 0.00$ & $\$ 0.00$ & \\
\hline 1. IMPRESIONES & & & & 500.000 & 500.000 & 0 & \\
\hline $\begin{array}{l}\text { APOYO DE } \\
\text { TRASNPORTE }\end{array}$ & & & & 500.000 & 500.000 & 0 & \\
\hline Imprevistos $(0 \%)$ & & & & $\$ 0.00$ & $\$ 0.00$ & $\$ 0.00$ & \\
\hline GRAN TOTAL & & & & 11.164 .000 & 6.712 .000 & - & \\
\hline
\end{tabular}




\section{Cronograma}

\begin{tabular}{|c|c|c|c|c|}
\hline \multicolumn{5}{|c|}{ Fecha del seguimiento } \\
\hline Actividad & No dias & Fecha Inicio & Fecha Finalización & Responsable \\
\hline \multicolumn{5}{|l|}{ PLANTEAMIENTO } \\
\hline Planteamiento de la Idea preliminar & 10 & $01 / 01 / 2013$ & $11 / 01 / 2013$ & Investigadores Principales \\
\hline Planteamiento de la pregunta de investigación & 5 & $12 / 01 / 2013$ & $17 / 01 / 2013$ & Investigadores Principales \\
\hline Revisión sistemática de la Literatura y Marco Teórico & 30 & $18 / 01 / 2013$ & $30 / 06 / 2013$ & Investigadores Principales \\
\hline \multicolumn{5}{|l|}{ PLANEACIÒN } \\
\hline Definición de la Hipotesis & 3 & $17 / 02 / 2013$ & $20 / 01 / 2013$ & Investigadores Principales \\
\hline Establecer las condiciones éticas del Estudio & 5 & $21 / 02 / 2013$ & $26 / 01 / 2013$ & Investigadores Principales \\
\hline Definición del Cronograma del proyecto & 5 & $27 / 02 / 2013$ & $01 / 02 / 2013$ & Investigadores Principales \\
\hline Establecer el presupuesto & 10 & $02 / 03 / 2014$ & $12 / 02 / 2014$ & Investigadores Principales \\
\hline Selección del diseño, Muestra, Control de sesgos & 5 & $13 / 03 / 2014$ & $18 / 02 / 2014$ & Investigadores Principales \\
\hline Diseño de Instrumentos de recolección de datos & 7 & $19 / 03 / 2014$ & $26 / 02 / 2013$ & Investigadores Principales \\
\hline Diseño de Protocolo de Investigación & 30 & $17 / 03 / 2015$ & $16 / 02 / 2013$ & Investigadores Principales \\
\hline \multicolumn{5}{|l|}{ APROBACIÓN Y VERIFICACIÓN } \\
\hline Aprobación docente de la universidad & 22 & $01 / 01 / 2015$ & $23 / 01 / 2013$ & Investigadores Principales \\
\hline Verificación por el Comité Técnico Científico & 30 & $02 / 04 / 2015$ & $22 / 02 / 2013$ & Integrantes de Comité \\
\hline Aprobación por comité de Investigaciones & 30 & $10 / 04 / 2013$ & $22 / 02 / 2013$ & Integrantes de Comité \\
\hline Elaboración del Acta Inicio & 10 & $10 / 04 / 2015$ & $04 / 03 / 2013$ & Hospitales Base \\
\hline Ajuste a la planeaciòn & 5 & $15 / 04 / 2015$ & $10 / 03 / 2013$ & Investigadores Principales \\
\hline \multicolumn{5}{|l|}{ IMPLEMENTACIÓN } \\
\hline Inicio del proyecto & - & $20 / 04 / 2015$ & $11 / 03 / 2013$ & Investigadores Principales \\
\hline \multicolumn{5}{|l|}{ Identificación de los $A B$ mas prescritos } \\
\hline Recolección de datos & 60 & $20 / 04 / 2015$ & $10 / 05 / 2013$ & Investigadores Principales \\
\hline \multicolumn{5}{|l|}{ REUNIONES DE TRABAJO DE SEGUIMIENTO } \\
\hline Reuniones de trabajo 1 & 15 & $30 / 05 / 2015$ & $26 / 03 / 2013$ & Investigadores Principales \\
\hline Reuniones de trabajo 2 & 15 & $30 / 07 / 2015$ & $11 / 04 / 2013$ & Investigadores Principales \\
\hline Reuniones de trabajo 3 & 15 & $30 / 09 / 2015$ & $27 / 04 / 2013$ & Investigadores Principales \\
\hline \multicolumn{5}{|l|}{ ANÁLISIS Y EVALUACIÓN } \\
\hline Interpretación de resultados & 30 & $01 / 10 / 2015$ & $12 / 01 / 2016$ & Investigadores Principales \\
\hline Análisis de datos & 30 & $01 / 10 / 2015$ & $12 / 12 / 2015$ & Investigadores Principales \\
\hline Análisis de resultados Final & 30 & $01 / 12 / 2015$ & $12 / 12 / 2015$ & Investigadores Principales \\
\hline Evaluación del proyecto & 10 & $05 / 02 / 2015$ & $23 / 02 / 2015$ & Investigadores Principales \\
\hline \multicolumn{5}{|l|}{ INFORME } \\
\hline Elaboración del informe Final & 15 & $01 / 02 / 2016$ & $04 / 05 / 2016$ & Investigadores Principales \\
\hline Presentación del informe Final & 15 & $20 / 01 / 2016$ & $05 / 02 / 2016$ & Investigadores Principales \\
\hline \multicolumn{5}{|l|}{ COMUNICACIÓN } \\
\hline Validación resultados por los integrantes Comité & 15 & $05 / 02 / 2016$ & $01 / 03 / 2015$ & Investigadores Principales \\
\hline Envío para publicación & 30 & $01 / 06 / 2016$ & $29 / 07 / 2016$ & Investigadores Principales \\
\hline
\end{tabular}




\section{Resultados}

Un total de 160 pacientes fueron incluidos en el estudio, 116 mujeres y 44 hombres, con una edad promedio de 38.6 años, los pacientes fueron clasificados según el consenso ARIA.

\section{(Tabla 1)}

\begin{tabular}{|c|c|}
\hline \multicolumn{2}{|c|}{ Características generales de la muestra } \\
\hline Número de pacientes & 160 \\
\hline Furoato de Mometasona & 80 \\
\hline Beclometasona & 73 \\
\hline Fluticasona & 7 \\
\hline Edad promedio & 38.3 \\
\hline Masculino & 44 \\
\hline Femenino & 116 \\
\hline Estrato socioeconomico promedio & 2 \\
\hline
\end{tabular}

\begin{tabular}{|c|c|c|}
\hline \multicolumn{3}{|c|}{ CLASIFICACIÓN CONSENSO ARIA } \\
\hline NÚMERO DE PACIENTES & LEVE & MODERADA - SEVERA \\
\hline 160 & 82 & 76 \\
\hline
\end{tabular}

Tabla 1. Características generales de la muestra.

El grupo de pacientes tratados con Beclometasona presentaba una puntuación global en el cuestionario ESPRINT-15 previo al tratamiento con beclometasona de 3.41 y posterior al tratamiento de $2.86 \mathrm{p}<0.000$. El grupo tratado con Mometasona presentaba una puntación global en el cuestionario ESPRINT-15 previo al tratamiento de 3.43 y posterior al tratamiento de $1.77(\mathrm{p}<0.000)$. (Tabla 2) 
No se encontró ninguna diferencia entre el puntaje del cuestionario ESPRINT-15 previo al tratamiento entre los dos grupos de tratamiento. Al comparar los puntajes entre ambos grupos posterior al tratamiento; los pacientes tratados con Mometasona mostraron mayor reducción en el cuestionario ESPRINT-15 con respecto a Beclometasona, esta reducción fue estadísticamente siginificativa (Beclometasona 3.01 vs Mometasona $2.08 \mathrm{p}<0.001$.

\begin{tabular}{|l|l|l|l|}
\hline Puntuación Global SPRINT 15 & Media & $\begin{array}{l}\text { Desv. } \\
\text { Estándar }\end{array}$ & P \\
\hline Beclometasona pre-tratamiento & 3,419178082 & 1,326346483 & \multirow{2}{*}{0,951} \\
\hline Furoato de mometasona - pre-tratamiento & 3,433333333 & 1,495835757 & \multirow{2}{*}{0,000} \\
\hline Beclometasona - post-tratamiento & 2,865753425 & 1,354894791 & \\
\hline $\begin{array}{l}\text { Furoato de mometasona - post- } \\
\text { tratamiento }\end{array}$ & 1,770464135 & 1,034270148 & \\
\hline
\end{tabular}

Tabla 2. Comparación de puntuación global ESPRINT-15.

Se evaluaron diferentes efectos adversos los cuales pueden ser observados en la tabla 2 . Dentro de los principales efectos adversos presentados en la población con el uso de Beclometasona fue la irritación de la nariz 43,80\%, dolor de cabeza 37,00\% y empeoramiento de los síntomas 35,60\% comparados con el tratamiento con Furoato de Mometasona el cual fue irritación de la nariz 10,0\%, dolor de cabeza 6,3\% y empeoramiento de los síntomas 8.8\%. Dentro de los pacientes sometidos bajo el tratamiento con fluticasona se ha podido observar que ninguno presenta como efecto adverso dolor de cabeza, el efecto adverso presentado generando síntomas leves fue mareos y estornudos posterior a la aplicación correspondiento al 57,10\%. (Tabla 3).

\begin{tabular}{|l|c|c|c|c|c|c|c|c|c|}
\hline \multirow{2}{*}{ Síntoma } & \multicolumn{3}{|c|}{ Beclometasona } & \multicolumn{2}{c|}{ Furoato de mometasona } & \multicolumn{3}{c|}{ Fluticasona } \\
\cline { 2 - 9 } & Leve & Moderado & Severo & Leve & Moderado & Severo & Leve & Moderado & Severo \\
\hline $\begin{array}{l}\text { Irritación o ardor en la } \\
\text { nariz }\end{array}$ & $21,90 \%$ & $21,90 \%$ & $43,80 \%$ & $43,80 \%$ & $23,80 \%$ & $10,00 \%$ & $14,30 \%$ & $14,30 \%$ & $14,30 \%$ \\
\hline Dolor de cabeza & $23,30 \%$ & $23,30 \%$ & $37,00 \%$ & $41,30 \%$ & $20,00 \%$ & $6,30 \%$ & $0,00 \%$ & $42,90 \%$ & $14,30 \%$ \\
\hline $\begin{array}{l}\text { Salida de agua por la } \\
\text { nariz }\end{array}$ & $30,10 \%$ & $28,80 \%$ & $28,80 \%$ & $40,00 \%$ & $23,80 \%$ & $8,80 \%$ & $28,60 \%$ & $14,30 \%$ & $14,30 \%$ \\
\hline $\begin{array}{l}\text { Alteraciones en el tono } \\
\text { de la voz }\end{array}$ & $24,70 \%$ & $24,70 \%$ & $30,10 \%$ & $35,00 \%$ & $23,80 \%$ & $6,30 \%$ & $42,90 \%$ & $14,30 \%$ & $0,00 \%$ \\
\hline $\begin{array}{l}\text { Aumento producción de } \\
\text { lágrimas }\end{array}$ & $21,90 \%$ & $24,70 \%$ & $30,10 \%$ & $35,00 \%$ & $18,80 \%$ & $11,30 \%$ & $14,30 \%$ & $14,30 \%$ & $14,30 \%$ \\
\hline
\end{tabular}




\begin{tabular}{|l|r|r|r|r|r|r|r|r|r|}
$\begin{array}{l}\text { Irritación o ardor de la } \\
\text { garganta }\end{array}$ & $21,90 \%$ & $28,80 \%$ & $31,50 \%$ & $38,80 \%$ & $12,50 \%$ & $11,30 \%$ & $42,90 \%$ & $14,30 \%$ & $14,30 \%$ \\
\hline $\begin{array}{l}\text { Estornudos después de } \\
\text { aplicar medicamento }\end{array}$ & $24,70 \%$ & $30,10 \%$ & $30,10 \%$ & $36,30 \%$ & $22,50 \%$ & $10,00 \%$ & $57,10 \%$ & $0,00 \%$ & $14,30 \%$ \\
\hline $\begin{array}{l}\text { Empeoramiento de los } \\
\text { síntomas }\end{array}$ & $17,80 \%$ & $21,90 \%$ & $35,60 \%$ & $33,80 \%$ & $16,30 \%$ & $8,80 \%$ & $28,60 \%$ & $14,30 \%$ & $0,00 \%$ \\
\hline Tos & $26,00 \%$ & $21,90 \%$ & $34,20 \%$ & $31,30 \%$ & $25,00 \%$ & $6,30 \%$ & $28,60 \%$ & $0,00 \%$ & $0,00 \%$ \\
\hline Mareo & $24,70 \%$ & $23,30 \%$ & $27,40 \%$ & $41,30 \%$ & $8,80 \%$ & $7,50 \%$ & $57,10 \%$ & $14,30 \%$ & $0,00 \%$ \\
\hline
\end{tabular}

Tabla 3. Efectos indeseables.

En general en la tabla que se muestra a continuación, podemos evidenciar las variables medidas con respecto a síntomas, actividades de la vida diaria, sueño y afectación psicológica con el uso de corticoides nasales para el tratamiento de la rinitis alérgica, esto es importante debido a que en Colombia existen múltiples tendencias de tratamiento para dicha patología, pero el tratamiento con corticoide nasal nos permite tener reducción en parámetros medidos que son relevantes en la calidad de vida de los pacientes, esto es muy importante en el momento de tomar decisiones antes de continuar con algún tipo de tratamiento. Se observa que en todas las variables evaluadas se encuentra una diferencia estadísticamente significativa $\mathrm{P}<0,000$.

\begin{tabular}{|l|r|r|r|r|r|r|c|}
\hline \multirow{2}{*}{ Variables } & \multirow{2}{*}{$\begin{array}{c}\text { Media Pre- } \\
\text { tratamiento }\end{array}$} & \multirow{2}{*}{$\begin{array}{c}\text { Media } \\
\text { Post- } \\
\text { tratamiento }\end{array}$} & \multirow{2}{*}{$\begin{array}{c}\text { Diferencia } \\
\text { Media }\end{array}$} & \multirow{2}{*}{$\begin{array}{c}\text { Desv. } \\
\text { Estándar }\end{array}$} & \multicolumn{2}{|c|}{$\begin{array}{c}\text { Intervalo de } \\
\text { confianza }\end{array}$} & \multirow{2}{*}{ P } \\
& Inferior & Superior & \\
\hline Síntomas & 3,58 & 2,506 & 1,0737 & 1,5603 & 0,8301 & 1,3174 & 0,000 \\
\hline $\begin{array}{l}\text { Activ. Vida } \\
\text { diaria }\end{array}$ & 3,119 & 2,100 & 1,0187 & 2,0457 & 0,6993 & 1,3382 & 0,000 \\
\hline Sueño & 3,731 & 2,273 & 1,4583 & 2,0838 & 1,133 & 1,7837 & 0,000 \\
\hline $\begin{array}{l}\text { Afectación } \\
\text { psicológica }\end{array}$ & 3,258 & 2,006 & 2,2613 & 0,1788 & 0,899 & 1,6052 & 0,000 \\
\hline
\end{tabular}

Tabla 4. Comportamiento de las variables evaluadas por el cuestrionario ESPRINT- 15 


\begin{tabular}{|l|r|r|r|c|}
\hline \multicolumn{4}{|c|}{ SÍNTOMAS } \\
\cline { 1 - 4 } Spray Nasal & \multicolumn{1}{|c|}{ Muestra } & \multicolumn{1}{|c|}{ Media } & \multicolumn{1}{c|}{$\begin{array}{c}\text { Desv. } \\
\text { Estándar }\end{array}$} & P \\
\cline { 1 - 4 } Beclometasona & 73,000 & 3,016 & 1,3403 & \multirow{2}{*}{0,00002} \\
\cline { 1 - 4 } Furoato de mometasona & 78,000 & 2,086 & 1,24007 & \\
\hline
\end{tabular}

\begin{tabular}{|l|r|r|r|c|}
\hline \multicolumn{4}{|c|}{ SUEÑO } \\
\hline Spray Nasal & \multicolumn{1}{|c|}{ Muestra } & \multicolumn{1}{c|}{ Media } & \multicolumn{1}{c|}{$\begin{array}{c}\text { Desv. } \\
\text { Estándar }\end{array}$} & P \\
\hline Beclometasona & 73,000 & 2,918 & 1,57212 & \multirow{2}{*}{0,00000} \\
\cline { 1 - 4 } Furoato de mometasona & 79,000 & 1,713 & 1,1944 & \\
\hline
\end{tabular}

\begin{tabular}{|l|r|r|r|c|}
\hline \multicolumn{5}{|c|}{ ACTIVIDAD VIDA DIARIA } \\
\hline Spray Nasal & \multicolumn{1}{|c|}{ Muestra } & Media & \multicolumn{1}{c|}{$\begin{array}{c}\text { Desv. } \\
\text { Estándar }\end{array}$} & P \\
\cline { 1 - 3 } Beclometasona & 73,000 & 2,772 & 1,7239 & \multirow{2}{*}{0,00001} \\
\cline { 1 - 4 } Furoato de mometasona & 79,000 & 1,608 & 1,36476 & \\
\hline
\end{tabular}

Tabla 5. Medición de calidad de vida evaluados en el cuestrionario ESPRINT-

\begin{tabular}{|l|r|r|r|c|}
\hline \multicolumn{4}{|c|}{ AFECTACIÓN PSICOLÓGICA } \\
\cline { 1 - 4 } Spray Nasal & \multicolumn{1}{|c|}{ Muestra } & \multicolumn{1}{c|}{ Media } & $\begin{array}{c}\text { Desv. } \\
\text { Estándar }\end{array}$ & P \\
\cline { 1 - 3 } Beclometasona & 73,000 & 2,772 & 1,69319 & \multirow{2}{*}{0,00000} \\
\cline { 1 - 3 } Furoato de mometasona & 79,000 & 1,367 & 1,07629 & \\
\hline
\end{tabular}

15

Posterior al tratamiento, se evidenció que el grupo tratado con Mometasona presentaba una reducción estadísticamente significativa en la severidad de síntomas ( 2.0 vs. 3.0, p<0.001), actividad diaria (1.6 vs. $2.7, \mathrm{p}<0.001)$, sueño (1.7 vs $2.9, \mathrm{p}<0.001)$ y afectación psicológica (1.3 vs 2.7, $\mathrm{p}<0.001)$ con respecto al grupo tratado con Beclometasona. (Tabla 4). Se observa una diferencia estadísticamente significativa respecto a la percepción calificada por el paciente antes y después del tratamiento con spray nasal al igual que en la puntuación Global ESPRINT 15. (Tabla 5) 


\section{Discusión}

La rinitis alérgica es una enfermedad común y prevalente que genera una importante carga socioeconómica y tiene un impacto significativo en la calidad de vida; afecta aproximadamente al 32\% de la población Colombiana $\left(^{2}\right)$. Con respecto a su tratamiento, los corticoides tópicos nasales son los medicamentos más efectivos y comunmente usados para el control de los síntomas en rinitis alérgica (Recomendación A) (7). En Colombia hay muy pocos estudios acerca de cuál es la afectación en la calidad de vida de los pacientes con rinitis alérgica tratados con corticoide nasal. Se conoce poco si existen diferencias en la calidad de vida de pacientes con diagnóstico clínico de rinitis alérgica tratados con Beclometasona, Furoato de Mometasona, Fluticasona.

En nuestro estudio encontramos que el grupo de pacientes tratados con Beclometasona presentaba una puntuación global en el cuestionario ESPRINT-15 previo al tratamiento con Beclometasona de 3.41 y posterior al tratamiento de $2.86 \mathrm{p}<0.000$. El grupo tratado con Furoato de Mometasona presentaba una puntación global en el cuestionario ESPRINT-15 previo al tratamiento de 3.43 y posterior al tratamiento de $1.77(\mathrm{p}<0.000)$. (Tabla 2) Al comparar los puntajes entre ambos grupos posterior al tratamiento; los pacientes tratados con Mometasona mostraron mayor reducción en el cuestionario ESPRINT-15 con respecto a Beclometasona, esta reducción fue estadísticamente siginificativa (Beclometasona 3.01 vs Mometasona $2.08 \mathrm{p}<0.001$. Se observa que en todas las variables evaluadas como: síntomas, actividades de la vida diaria, sueño y afectación psicológica se encuentra una diferencia estadísticamente significativa $\mathrm{P}<0,000$.

Dentro de los principales efectos adversos presentados en la población con el uso de Beclometasona fue la irritación de la nariz 43,80\%, dolor de cabeza 37,00\% y empeoramiento de los síntomas 35,60\% comparados con el tratamiento con Furoato de 
Mometasona el cual fue irritación de la nariz 10,0\%, dolor de cabeza 6,3\% y empeoramiento de los síntomas $8.8 \%$.

Numerosos estudios se han realizado para comprobar por qué la beclometasona en spray nasal es eficaz para el tratamiento de la rinitis alérgica. Meltzer et. al realizaron un estudio para evaluar los efectos adversos la eficacia y seguridad del tratamiento de rinitis alérgica con rinitis perenne, comparando el tratamiento con beclometasona Vs placebo, demostrando la superioridad en mejoría de síntomas y reporte de efectos adversos; se observa que el efecto adverso de disconfort nasal comparado con el placebo tiene un índice de porcentaje mayor en presentación. Este estudio no se podría extrapolar a nuestra población debido a que en Colombia no existen las estaciones, si se puede afirmar como ya se conoce en los estudios realizados que el uso de corticoides nasales para el manejo de la rinitis alérgica mejora parámetros de calidad de vida como se muestra en la (Tabla 4).

El Furoato de Mometasona es un corticosteroide intranasal que tiene una serie de cualidades que son importantes en la consecución de la selectividad nasal con efectos adversos sistémicos mínimos; se ha comprobado que los perfiles de eficacia y seguridad en el uso clínico son consistentes con su propiedades farmacocinéticas y farmacodinámicas (11). En el mercado, el precio del Furoato de Mometasona es mayor comparado con el de la beclometasona; pero cuando se suman todos los efectos adversos presentados con el uso de la beclometasona como por ejemplo: número de reconsultas, pago por tratamientos adicionales, inconformidad del paciente con el tratamiento instaurado, estos costos adicionales compensan el valor de ahorro con el uso de la Beclometasona (9)

Dentro de los efectos adversos locales observados por el uso de los mismos se ha encontrado, la irritación nasal o epistaxis. $\left(^{7}\right)$. Con respecto a los efectos indeseables evaluados en comparación con los dos tratamientos podemos observar que los ítems calificados por el cuestionario en el grupo tratado con Beclometasona comparado con el grupo tratado con Furoato de Mometasona, mostró un comportamiento superior, con una diferencia estadísticamente significativa (Tabla 2). Dentro de este modelo se demostró que los efectos adversos presentados por los pacientes con el uso de la beclometasona son mayores y requieren costos adicionales de tratamiento con respecto al uso de Furoato de Mometasona. El análisis anteriormente mencionado muestra que en Colombia, el uso de 
Furoato de Mometasona para el tratamiento de rinitis alérgica mostró una mayor mejoría en la sintomatología de los pacientes, esto refleja tanto la eficacia como la seguridad a menores costos totales de tratamiento. (9)

En Colombia, el tratamiento con corticoides nasales para pacientes con rinitis alérgica, está limitado al único medicamento incluído en el Plan Obligatorio de Salud (Beclometasona), la experiencia de cada profesional y a su preferencia a determinada molécula. Los pacientes que no responden al tratamiento o que presentan reacciones indeseables con el uso de la Beclometasona, le son formulados corticoides nasales como la Fluticasona, Mometasona, Budesonida, Triamcinolona o la Ciclesonida, los cuales no están incluidos en el POS, y por lo tanto los costos de estos medicamentos son asumidos por el paciente. Los costos para el tratamiento de rinitis alérgica y los costos indirectos relacionados a las alteraciones en productividad laboral como resultado de la enfermedad son sustanciales. El costo estimado total directo ( 7.3 billones de dólares) e indirecto ( 4.28 billones de dólares) para los Estados Unidos de América en el 2002, siendo el total de 11.58 billones $\left({ }^{7}\right)$. En un estudio realizado en Colombia referente a un modelo de toma de análisis para estimar la costo-efectividad del furoato de mometasona en comparación con el dipropionato de beclometasona para el tratamiento de pacientes pediátricos con rinitis alérgica durante un período de 12 meses. Se demostró que el tratamiento con Furoato de mometasona comparado con el tratamiento de beclometasona se asoció con menores costos (US \$ 229.78 vs. 289,74 costo medio por paciente de más de 12 meses).

En la actualidad, a pesar de las múltiples opciones de medicamentos disponibles en el mercado para el tratamiento de la rinitis alérgica, se ha logrado demostrar que sigue afectando sustancialmente la calidad de vida del paciente (10). Esta es una de las motivaciones para realización de nuestro estudio, ya que a pesar de someter a los pacientes a cualquier tipo de medicación, lo que hay que tener en cuenta como especialistas es la percepción que tenga el paciente con respecto a su tratamiento y como éste ha influido en su calidad de vida.

Las debilidades encontradas en nuestro estudio consistieron en que no fue un estudio doble ciego pero aleaorizado; por deficiencias administrativas con respecto a la entrega de medicamentos NO POS no se logro obtener una muestra representativa de pacientes 
tratados con Fluticasona lo que no permitio su comparación con los otros corticoides nasales. Una de nuestras fortalezas consistió en que ninguno de los pacientes incluidos en nuestro estudio recibieron otro tipo de medicación para el tratamiento lo cual permite evaluar la efectividad del corticoide nasal. Teniendo en cuenta que toda nuestra población evaluada asistia a consulta externa del Hospital Universitario La Samaritana, realizamos a aleatorización de los mismos para evitar el sesgo de selección.

Encontramos que los pacientes con rinitis alérgica tratados con Furoato de Mometasona presentaban una mejoría estadísticamente significativa en la calidad de vida analizada mediante el cuestionario ESPRINT-15 en comparación a los pacientes tratados con Beclometasona.

La Mometasona comparado con la Beclometasona, muestra ser más potente en mejorar los puntajes de calidad de vida medidos mediante el cuestionario SPRINT-15 en pacientes con Rinitis Alérgica. Adicionalmente, la Mometasona mostró menos efectos adversos comparado a la Beclometasona mejorando aún más la calidad de vida en pacientes con Rinitis alérgica, sin embargo, se requiere más estudios con mayor seguimiento. 


\section{Conclusiones}

La rinitis alérgica es una enfermedad altamente prevalente en Colombia la cual usualmente se trata con corticoides tópicos nasales. La Beclometasona y Furoato de Mometasona demostraron ser medicamentos efectivos para el tratamiento de la rinitis alérgica; de estos dos, el Furoato de mometasona demostró ser más efectiva que la beclometasona en el control de los sintomas relacionados a síntomas, actividades de la vida diaria, sueño y afectación psicológica.

De otra manera, la Beclometasona demostró mayor frecuencia de efectos adversos con respecto al uso de el Furoato de mometasona, sin embargo, consideramos que se necesitan más estudios, especialmente multi-instuticionales doble ciegos y aleatorizados que incluyan diferentes corticoides nasales. 


\section{Recomendaciones}

En la actualidad existen una gran variedad de tratamientos para la rinitis alérgica, debido a la alta prevalencia de esta patología en nuestro país. Recomendamos que dentro de las decisiones clínicas para el uso de cualquier tratamiento con corticoide nasal se tenga en cuenta la percepción y la respuesta con respecto a su calidad de vida evaluada por el paciente. Estos resultados pueden ayudar a apoyar la toma de decisiones clínicas hasta que la evidencia más robusta esté disponible. 


\section{Referencias bibliográficas}

1. Bousquet J, Khaltaev N, Cruz AA, et al. Allergic Rhinitis and its Impact on Asthma (ARIA) 2008 update (in collaboration with the World Health Organization, GA(2)LEN and AllerGen). Allergy. 2008;63 Suppl 86:8-160. doi:10.1111/j.1398-9995.2007.01620.x.

2. Dennis RJ, Caraballo L, García E, et al. Prevalence of asthma and other allergic conditions in Colombia 2009-2010: a cross-sectional study. BMC Pulm Med. 2012;12:17. doi:10.1186/1471-2466-12-17.

3. Mygind N, Andersson M. Topical glucocorticosteroids in rhinitis: clinical aspects. Acta Otolaryngol. 2006;126(10):1022-1029. doi:10.1080/00016480600672550.

4. Mullol J, Obando A, Pujols L, Alobid I. Corticosteroid treatment in chronic rhinosinusitis: the possibilities and the limits. Immunol Allergy Clin North Am. 2009;29(4):657-668. doi:10.1016/j.iac.2009.07.001.

5. Ait-Khaled N, Pearce N, Anderson HR, et al. Global map of the prevalence of symptoms of rhinoconjunctivitis in children: The International Study of Asthma and Allergies in Childhood (ISAAC) Phase Three. Allergy. 2009;64(1):123-148. doi:10.1111/j.13989995.2008.01884.x. 
6. Rabe KF, Adachi M, Lai CKW, et al. Worldwide severity and control of asthma in children and adults: the global asthma insights and reality surveys. J Allergy Clin Immunol. 2004;114(1):40-47. doi:10.1016/j.jaci.2004.04.042.

7. Wallace DV, Dykewicz MS, Bernstein DI, et al. The diagnosis and management of rhinitis: an updated practice parameter. J Allergy Clin Immunol. 2008;122(2 Suppl):S1-84. doi:10.1016/j.jaci.2008.06.003.

8. Valero A, Baró E, Sastre J, et al. Reference values for facilitating the interpretation of the ESPRINT-15 questionnaire (Spanish version). $J$ Investig Allergol Clin Immunol. 2009;19(5):396-403.

9. Martínez C, Sossa M, Lemos E. Cost-Effectiveness Analysis of Mometasone Furoate Versus Beclomethasone Dipropionate for the Treatment of Pediatric Allergic Rhinitis in Colombia. Adv Ther 2015; 32:254-269.

10. Berger W, Meltzer E. Intranasal spray medications for maintenance therapy of allergic rhinitis. Am J Rhinol Allergy 2015; 29: 273-282.

11. Berlucchi M, Pedruzzi B. Intranasal Mometasone Furoate for Treatment of Allergic Rhinitis. Clinical Medicine Insights: Therapeutics 2010:2 761-769

12. Yonezaki M, Akiyama K, Karaki M, Goto R, et. all. Preference evaluation and perceived sensory comparison of fluticasone furoate and mometasone furoate intranasal sprays in allergic rhinitis. Auris Nasus Larynx 2015.

13. Penagos M, Compalati M, Tarantini F, et.all. Efficacy of mometasone furoate nasal spray in the treatment of allergic rhinitis. Meta-analysis of randomized, double-blind, placebo-controlled, clinical trials. Allergy 2008: 63: 1280-1291.

14. Meltzer E, Jacobs E, LaForce C, Kelle L, et.all. Safety and efficacy of once-daily treatment with beclomethasone dipropionate nasal aerosol in subjects with perennial allergic rhinitis. Allergy Asthma Proc 2012; 33:249 -257.

15. Farid R, Farid F, Ghaffari J, Jabbarri F. Evaluation of fluticasone nasal spray vs 
Beclometasona nasal spray in the treatment of allergic rhinitis. Iranian journal of allergy, asthma and inmunology. 2003; 2: 193- 196.

16. Jaruvongvanich V, Mongkolpathumrat P, Chantaphakul H, Klaewsongkram J. Extranasal symptoms of allergic rhinitis are difficult to treat and affect quality of life. 2015; $1-5$.

17. Blaiss MS, Safety update regarding intranasal corticosteroids for the treatment of allergic rhinitis. Allergic asma proc. 2011 Nov-Dec;32(6):413-8.

18. Berger, W; Jacobs R; Amar N ; Sudeesh K, et all. Efficacy and safety of beclomethasone dipropionate nasal aerosol in children with perennial allergic rhinitis. Ann Allergy Asthma Immunol 115 (2015) 130-136.

19. Crawford1 B, Stanford R, Wong A, Dalal A, Bayliss M. Psychometric validation of the experience with allergic rhinitis nasal spray questionnaire. Patient Related Outcome Measures 2011:2 127-133.

20. Piotr K; Wojciech; J, Spencer K, Katarina Z. Comparative safety and efficacy of two formulations of mometasone nasal spray in adult seasonal allergic rhinitis. Allergy and asthma procedingns 2014, Volume 35, Number 4: 332-337(6).

21. Davies R, Nelson H. Once-Daily Mometasone Furoate Nasal Spray: Efficacy and Safety of a New Intranasal Glucocorticoid for Allergic Rhinitis. Clinical therapeutics. 1997; 19: 27-38.

22. Hebert JR, Nolop K, Lutsky B. Once daily mometasone furoate aqueous nasal spray (Nasonex) in seasonal allergic rhinitis: An active- and placebo-controlled study. Allergy. $1996 ; 51: 569-576$.

23. Drouin M, Yang WH, Bertrand B, et al. Once daily mometasone furoate aqueous nasal spray is as effective as twice daily beclomethasone dipropionate for perennial allergic rhinitis patients. Ann Allergy Asthma Zmmunol. 1996:77:153-160. 
24. Sharpe M, Jarvis B. Inhaled mometasone furoate: A review of its use in adults and adolescents with persistent asthma [published correction appears in Drugs. 2001;61:13251350.

25. Lipworth BJ, Jackson CM. Safety of inhaled and intranasal corticosteroids: Lessons for the new millennium. Drug Saf. 2000;23:11-33.

26. Davies RJ, Nelson HS. Once-daily mometasone furoate nasal spray: Efficacy and safety of a new intranasal glucocorticoid for allergic rhinitis. Clin Ther. 1997;19:27-38; discussion 2-3.

27. Frieri M, Therattil J, Chavarria V, et al. Effect of mometasone furoate on early and late phase inflammation in patients with seasonal allergic rhinitis. Ann Allergy Asthma Immunol. 1998;81:431-437.

28. Trangsrud AJ, Whitaker AL, Small RE. Intranasal corticosteroids for allergic rhinitis. Pharmacotherapy. 2002;22:1458- 1467.

29. Gawchik S, Goldstein S, Prenner B, John A. Relief of cough and nasal symptoms associated with allergic rhinitis by mometasone furoate nasal spray. Ann Allergy Asthma Immunol. 2003;90:416-421.

30. Austin RJ, Maschera B, Walker A, et al. Mometasone furoate is a less specific glucocorticoid than fluticasone propionate. Eur Respir J. 2002; 20:1386-1392. 
Anexos

Anexo 1.

Datos de Ingreso y Cuestionario SPRINT-15 Validado al español.

Nombre:

Fecha:

\section{Edad: ___ Sexo: ___ Teléfono de contacto:}

Dirección de Residencia:

Estrato:

Inhalador Nasal:

Dosis:

Frecuencia: 
Seguidamente, encontrará algunas preguntas sobre las molestias ocasionadas por la rinitis. Por favor, rodee con un círculo el número correspondiente a la respuesta que Usted elija. Es importante que conteste a todas las preguntas.

\begin{tabular}{|c|c|c|c|c|c|c|c|}
\hline \multicolumn{8}{|c|}{ Durante las últimas 2 semanas, ¿cuánto le ha molestado cada uno de los siguientes síntomas? } \\
\hline Síntomas & $\begin{array}{l}\text { No me ha } \\
\text { molestado } \\
\text { nada }\end{array}$ & $\begin{array}{c}\text { No me ha } \\
\text { molestado casi } \\
\text { nada }\end{array}$ & $\begin{array}{l}\text { Me ha } \\
\text { molestado } \\
\text { poco }\end{array}$ & $\begin{array}{l}\text { Me ha molestado } \\
\text { moderadamente }\end{array}$ & $\begin{array}{l}\text { Me ha } \\
\text { molestado } \\
\text { bastante }\end{array}$ & $\begin{array}{c}\text { Me ha } \\
\text { molestado } \\
\text { mucho }\end{array}$ & $\begin{array}{c}\text { Me ha } \\
\text { molestado } \\
\text { muchísimo }\end{array}$ \\
\hline $\begin{array}{l}\text { 1. La sensación de tener la nariz tapada } \\
\text { o la sensación de obstrucción }\end{array}$ & 0 & 1 & 2 & 3 & 4 & 5 & 6 \\
\hline $\begin{array}{l}\text { 2. La mucosidad nasal líquida o como } \\
\text { agua }\end{array}$ & 0 & 1 & 2 & 3 & 4 & 5 & 6 \\
\hline $\begin{array}{l}\text { 3. El picor en la nariz o estornudos } \\
\text { repetidos }\end{array}$ & 0 & 1 & 2 & 3 & 4 & 5 & 6 \\
\hline $\begin{array}{l}\text { 4. El picor de ojos o tener que rascarse } \\
\text { los ojos }\end{array}$ & 0 & 1 & 2 & 3 & 4 & 5 & 6 \\
\hline $\begin{array}{l}\text { 5. La dificultad para respirar, la } \\
\text { sensación de asfixia o ahogo }\end{array}$ & 0 & 1 & 2 & 3 & 4 & 5 & 6 \\
\hline
\end{tabular}

\begin{tabular}{|c|c|c|c|c|c|c|c|}
\hline \multicolumn{8}{|c|}{ Durante las últimas 2 semanas, ¿cuánto le ha molestado cada una de las siguientes situaciones? } \\
\hline Actividades de la vida diaria & $\begin{array}{l}\text { No me ha } \\
\text { molestado } \\
\text { nada }\end{array}$ & $\begin{array}{c}\text { No me ha } \\
\text { molestado casi } \\
\text { nada }\end{array}$ & $\begin{array}{c}\text { Me ha } \\
\text { molestado } \\
\text { poco }\end{array}$ & $\begin{array}{l}\text { Me ha molestado } \\
\text { moderadamente }\end{array}$ & $\begin{array}{c}\text { Me ha } \\
\text { molestado } \\
\text { bastante }\end{array}$ & $\begin{array}{c}\text { Me ha } \\
\text { molestado } \\
\text { mucho }\end{array}$ & $\begin{array}{c}\text { Me ha } \\
\text { molestado } \\
\text { muchisimo }\end{array}$ \\
\hline $\begin{array}{l}\text { 6. La incomodidad o dificultad para } \\
\text { trabajar, a causa de la rinitis }\end{array}$ & 0 & 1 & 2 & 3 & 4 & 5 & 6 \\
\hline $\begin{array}{l}\text { 7. Los síntomas de la rinitis cenando o } \\
\text { tomando algo fuera de casa }\end{array}$ & 0 & 1 & 2 & 3 & 4 & 5 & 6 \\
\hline $\begin{array}{l}\text { 8. Interrumpir constantemente lo que } \\
\text { estaba haciendo, a causa de la rinitis }\end{array}$ & 0 & 1 & 2 & 3 & 4 & 5 & 6 \\
\hline
\end{tabular}

\begin{tabular}{|c|c|c|c|c|c|c|c|}
\hline Sueño & $\begin{array}{c}\text { No me ha } \\
\text { molestado } \\
\text { nada }\end{array}$ & $\begin{array}{c}\text { No me ha } \\
\text { molestado casi } \\
\text { nada }\end{array}$ & $\begin{array}{c}\text { Me ha } \\
\text { molestado } \\
\text { poco }\end{array}$ & $\begin{array}{l}\text { Me ha molestado } \\
\text { moderadamente }\end{array}$ & $\begin{array}{c}\text { Me ha } \\
\text { molestado } \\
\text { bastante }\end{array}$ & $\begin{array}{c}\text { Me ha } \\
\text { molestado } \\
\text { mucho }\end{array}$ & $\begin{array}{c}\text { Me ha } \\
\text { molestado } \\
\text { muchisimo }\end{array}$ \\
\hline $\begin{array}{l}\text { 9. Los problemas para dormir o las } \\
\text { dificultades para conciliar el sueño, a } \\
\text { causa de la rinitis }\end{array}$ & 0 & 1 & 2 & 3 & 4 & 5 & 6 \\
\hline $\begin{array}{l}\text { 10. Levantarse con sequedad en la boca } \\
\text { o despertarse por esto, a causa de la } \\
\text { rinitis }\end{array}$ & 0 & 1 & 2 & 3 & 4 & 5 & 6 \\
\hline 11. Dormir mal, a causa de la rinitis & 0 & 1 & 2 & 3 & 4 & 5 & 6 \\
\hline
\end{tabular}

\begin{tabular}{|c|c|c|c|c|c|c|c|}
\hline Afectación psicológica & $\begin{array}{c}\text { No me ha } \\
\text { molestado } \\
\text { nada }\end{array}$ & $\begin{array}{c}\text { No me ha } \\
\text { molestado casi } \\
\text { nada }\end{array}$ & $\begin{array}{l}\text { Me ha } \\
\text { molestado } \\
\text { poco }\end{array}$ & $\begin{array}{l}\text { Me ha molestado } \\
\text { moderadamente }\end{array}$ & $\begin{array}{c}\text { Me ha } \\
\text { molestado } \\
\text { bastante }\end{array}$ & $\begin{array}{l}\text { Me ha } \\
\text { molestado } \\
\text { mucho }\end{array}$ & $\begin{array}{c}\text { Me ha } \\
\text { molestado } \\
\text { muchisimo }\end{array}$ \\
\hline $\begin{array}{l}\text { 12. Tener que estar pendiente de la } \\
\text { rinitis }\end{array}$ & 0 & 1 & 2 & 3 & 4 & 5 & 6 \\
\hline $\begin{array}{l}\text { 13. Estar más irritable o de mal humor, a } \\
\text { causa de la rinitis }\end{array}$ & 0 & 1 & 2 & 3 & 4 & 5 & 6 \\
\hline $\begin{array}{l}\text { 14. Pasarlo mal o sentirse mal, a causa } \\
\text { de la rinitis }\end{array}$ & 0 & 1 & 2 & 3 & 4 & 5 & 6 \\
\hline \multicolumn{8}{|c|}{ 15. En general, teniendo en cuenta su rinitis y ningún otro trastorno, ¿cómo diría que es su salud? } \\
\hline$\square$ Excelente & $\square$ Muy Buena & \multicolumn{2}{|c|}{$\square$ Buena } & \multicolumn{2}{|c|}{$\square$ Regular } & \multicolumn{2}{|c|}{$\square$ Mala } \\
\hline
\end{tabular}


Anexo 2.

Datos de Seguimiento, Efectos Adversos y Cuestionario ESPRINT-15 Validado al español.

Nombre:

Fecha:

Edad:

Sexo:

Teléfono de contacto:

Dirección de Residencia:

Estrato:

Inhalador Nasal:

Dosis: Frecuencia:

Efectos Adversos - Marque de acuerdo a la severidad de los síntomas como:

0. Nada, 1. Leve, 2. Moderado, 3. Severo

Irritación o Ardor de la Nariz:

Irritación o Ardor de la Garganta:

Tos:

Dolor de Cabeza:

Sangrado por la Nariz:

Mareo:

Salida de agua por la nariz: 
Estornudos después de aplicar el medicamento:

Alteraciones en el tono de la voz:

Empeoramiento de los síntomas:

Aumento en la producción de lágrimas:

Diligencie de nuevo el cuestionario de acuerdo a su sintomatología actual, después de haber usado el inhalador nasal por lo menos por 3 meses.

Seguidamente, encontrará algunas preguntas sobre las molestias ocasionadas por la rinitis. Por favor, rodee con un círculo el número correspondiente a la respuesta que Usted elija. Es importante que conteste a todas las preguntas.

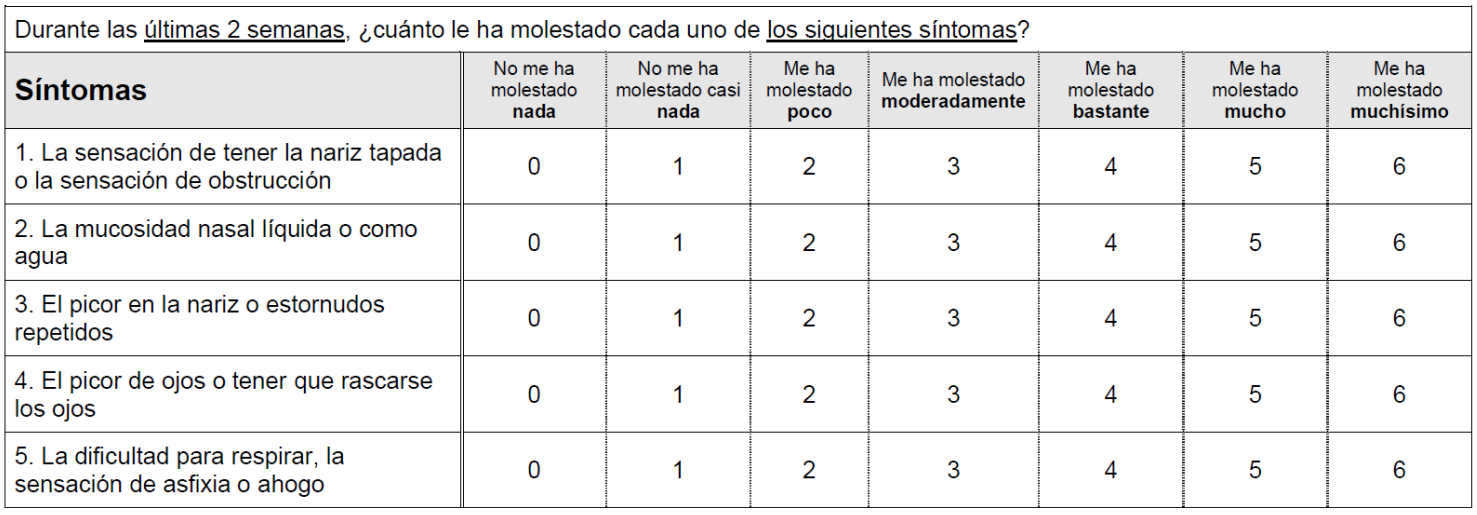

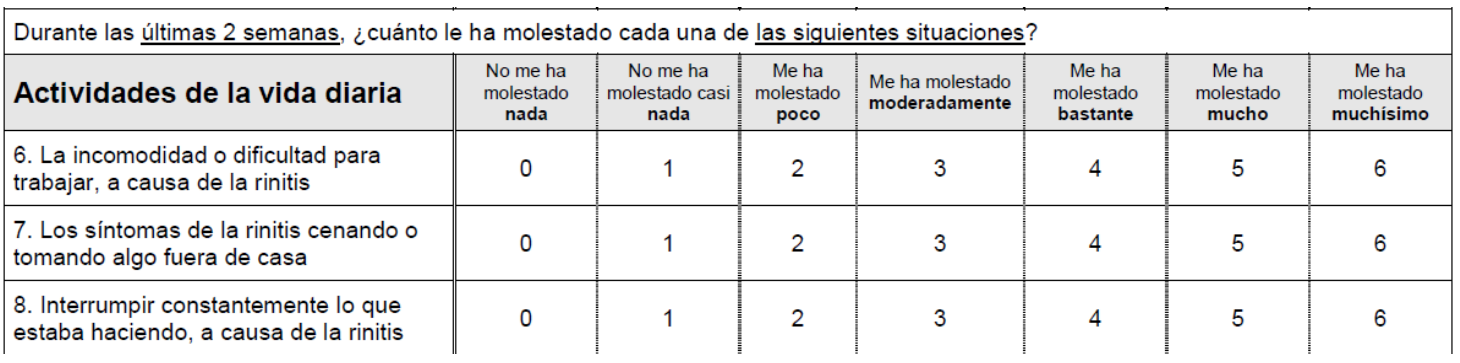

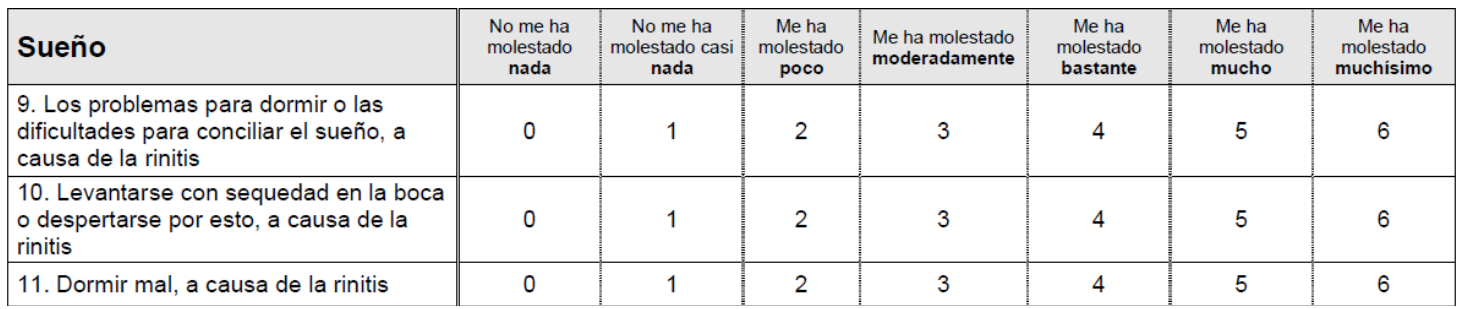




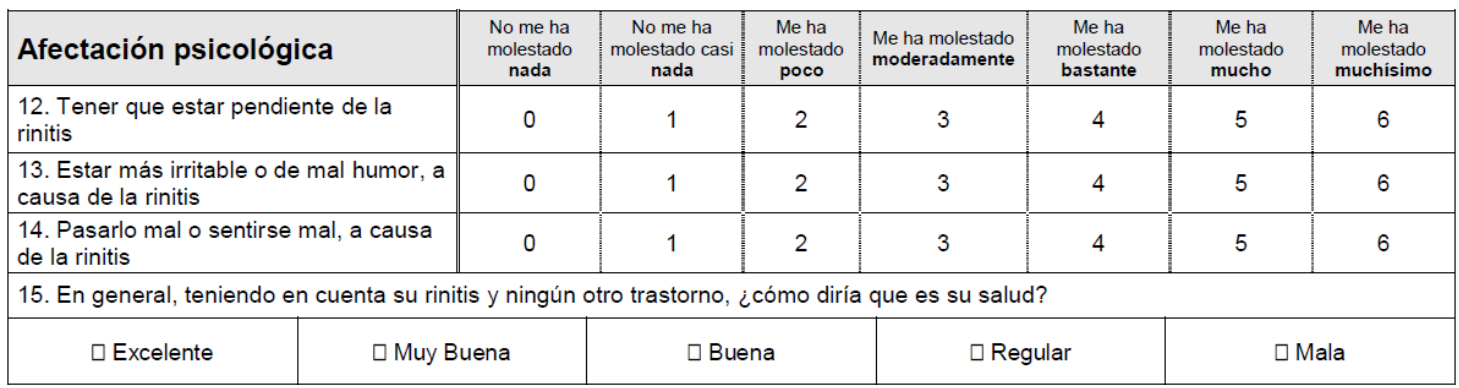




\section{Anexo 3. Metodología para el cálculo de los resultados del cuestionario ESPRINT-15}

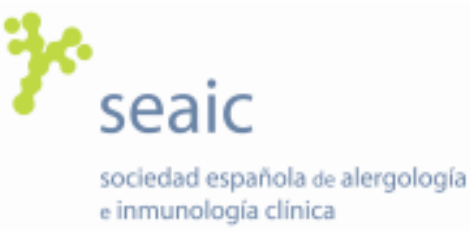

\section{METOdología PARA EL CÁLCULO dE RESULTADOS DEL LOS CUESTIONARIOS \\ ESPRINT-15 y ESPRINT-28}

Puntuación de cada una de las dimensiones:

Tanto para ESPRINT-15 como ESPRINT-28 se realiza un promedio de la puntuación dada a cada uno de los ítemes ( 0 a 7$)$ de ese dominio concreto.

P.ej.: Puntuación del dominio de síntomas

$=\Sigma$ de puntuación de cada ítem $/ 5$

\section{Puntuación global:}

Tanto para ESPRINT-15 como ESPRINT-28, se realiza la suma global de las puntuaciones (que oscilan de 0 a 6 ) de los 14 ítems (ESPRINT-15) o de los 27 items (ESPRINT-28) más la puntuación dada en el cuestionario general (que oscila de 0 a 4 : Excelente $=0$, Muy buena $=1$, Buena $=2$, Regular $=3$, Mala $=4$.) Se divide dicha suma por el número total de items (15 en ESPRINT-15 y 28 en ESPRINT-28).

\section{Interpretación de los resultados:}

- La puntuación por dimensiones oscila entre 0 (bajo impacto en la CVRS) y 6 (alto impacto en la CVRS)

- La puntuación global oscila entre 0 (bajo impacto en la CVRS) y 5,8 (alto impacto en la CVRS). 


\title{
DEPARTAMENTO DE OTORRINOLARINGOLOGIA
}

\author{
Grupo de Investigación
}

PROYECTO:

Calidad de Vida en pacientes con Rinitis Alérgica bajo tratamiento con Corticoide Tópico Nasal.

INVESTIGADORES: Dr. Camilo Andrés Reyes Gelves - Dra. Lilian Andrea

Ballesteros Rodríguez

TUTOR TEMÁTICO: Dr. Omar Gutiérrez - Dr. Luis Jorge Mejía

TUTOR METODOLÓGICO: Dra. Lina Sofia Morón

\section{CONSENTIMIENTO INFORMADO}

Día/mes/año: /

Código del Sujeto:

Este documento explica en qué consiste este estudio de investigación. Es importante que usted lea cuidadosamente esta información y que luego decida si desea participar o no como voluntario.

\section{PROPÓSITO:}

El propósito de esta investigación es determinar la calidad de vida mediante el cuestionario ESPRINT-15 validado al español en pacientes con diagnostico de Rinitis Alérgica sin tratamiento y tratados con corticoide nasal tópico por un periodo mínimo de 3 meses. 


\section{DURACIÓN:}

El tiempo que cada sujeto de estudio otorgará para esta investigación será tan sólo 10 minutos mientras diligencia el cuestionario junto a otra información general. El tiempo disponible para la recolección y análisis de los datos es de 6 meses. Los resultados del estudio se reportarán en Enero de 2014.

\section{PROCEDIMIENTOS:}

Los procedimientos que le conciernen a usted como sujeto en este estudio incluyen:

- Responder unas preguntas con el fin de realizar una historia clínica.

- Responder el cuestionario ESPRINT-15 junto a datos generales.

- Responder la presencia o ausencia de efectos indeseables secundario al uso del corticoide nasal.

\section{POSIBLES RIESGOS O INCOMODIDADES:}

No se prevé ningún riesgo serio para los sujetos en este proyecto de investigación. Los posibles riesgos o incomodidades asociadas con su participación en este estudio incluyen:

- Efectos adversos secundarios al uso de alguno de los corticoides nasales Beclometasona, Furoato de Mometasona, Fluticasona o Budesonida como Irritación o Ardor de la Nariz, Irritación o Ardor de la Garganta, Tos, Dolor de Cabeza, Sangrado de la Nariz, Mareo, Salida de agua por la nariz, Estornudos después de aplicar el medicamento, Alteraciones en el tono de la voz, Empeoramiento de los síntomas y Aumento en la producción de lágrimas.

\section{BENEFICIOS:}

- Determinar está afectada su calidad de vida por la Rinitis.

- Determinar cómo se altera su calidad de vida mediante el tratamiento con corticoide nasal tópico. 


\section{CONFIDENCIALIDAD:}

Se realizará todo esfuerzo para asegurar que los hallazgos de este estudio sean confidenciales. Copias de los documentos de este estudio serán conservados en el archivo del Comité de Investigaciones del Hospital Universitario de La Samaritana por un período mínimo de tres años. Los cuestionarios diligenciados serán desechados al finalizar el estudio y no podrán ser utilizados para ningún análisis diferente al propuesto. Los hallazgos de este estudio podrán ser publicados o presentados en reuniones sin revelar su nombre o identidad. Se protegerán sus derechos y privacidad; no obstante los investigadores asociados con esta investigación tendrán acceso a los documentos e información relacionados con este estudio. Su expediente será completamente confidencial según lo indica la Ley. Este no será compartido con otras personas a menos que sea exigido por la Ley o de acuerdo con las especificaciones mencionadas anteriormente.

\section{PARTICIPACION VOLUNTARIA:}

Su participación en este estudio de investigación es absolutamente voluntaria, por lo tanto, no habrá ningún tipo de remuneración económica o compensación por su participación, excepto poder determinar su calidad de vida. Usted puede rehusarse a participar o puede retirarse del estudio en cualquier momento que lo desee después de haber ingresado (llamando al Doctora Lilian Andrea Ballesteros Rodríguez al teléfono 3188482117 o al Dr. Omar Gutiérrez al teléfono 310-4851514). Se le informará inmediatamente si cualquier hallazgo de este estudio podría tener impacto en su salud.

Su firma confirma que ha leído y comprendido este documento. En caso de participar, recibirá una copia firmada de este consentimiento informado. A usted se le ha dado y se le continuará dando la oportunidad de hacer preguntas y discutir con los investigadores su participación.

- Usted ha elegido PARTICIPAR en este proyecto de investigación libre y voluntariamente y en constancia firma el presente documento: 
Día/mes/año:

\section{FIRMA DEL SUJETO VOLUNTARIO}

Día/mes/año:

FIRMA DE TESTIGO

Día/mes/año:

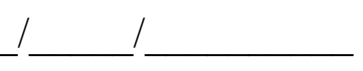

FIRMA DE TESTIGO

Día/mes/año:

\section{FIRMA DEL INVESTIGADOR}

Usted ha decidido NO participar en este proyecto de investigación y en constancia firma:

Día/mes/año:

\section{FIRMA DEL SUJETO VOLUNTARIO}

